\title{
INTERNATIONAL SYMPOSIUM ON CONCUSSION IN SPORT
}

\author{
The International Ice Hockey Federation (IIHF), in partnership with FIFA, the Federation Internationale de Football Association Medical \\ Assessment and Research Center (F-MARC), and the International Olympic Committee Medical Commission (IOC) is organising an Inter- \\ national Symposium on Concussion in Sport. \\ Faced with the increasing incidence of this potentially dangerous injury in ice hockey and other sports, we have gathered the leading medi- \\ cal experts from all parts of the world and from a wide sampling of different sports, experienced in dealing with sports related head injuries. \\ Our objective is to understand, as completely as possible, what actually takes place when severe blows to the head occur. It is our hope, that \\ with the information learned, we can begin educating our athletes with the goal of eliminating concussions in all sports. \\ We are most grateful to the esteemed presenters for their extraordinary efforts, and to the many others who have submitted abstracts and \\ papers for consideration.

\section{Welcome from the Scientific Committee}

On behalf on the Scientific Program Committee I would like to welcome all delegates to the International Symposium on Concussion in Sport. This Symposium has been sponsored by the International Ice Hockey Federation (IIHF) in cooperation with the Federation Internationale de Football Association Medical Assessment and Research Centre (F-MARC) and the International Olympic Committee Medical Commission (IOC).

The IIHF has chosen Vienna as the host city for this meeting.

The aim of the Symposium is to provide recommendations addressing this important topic for the improvement of safety and health of athletes in all sporting fields who suffer concussive injuries. To this end, the Committee has invited a range of speakers, all eminent in their fields, to address specific issues of epidemiology, clinical science, protective equipment, cognitive assessment, and long term outcome. The faculty represents the most important gathering of "concussionologists" yet seen in sports medicine.

In addition, research poster presentations have been invited and will demonstrate the range of concussion research currently

\section{PROBABILITY OF CONCUSSION FROM SINGLE HEADING EVENTS IN FOOTBALL}

C. Barr, M. Beusenberg. Biokinetics and Associates Ltd, Ottawa, Ontario, Canada.

Scope: This pilot study takes a look at the severity levels of a football impact against the head while in different orientations relative to the ball trajectory. Biomechanical knowledge of the tolerance of the brain to concussion as a result of direct and indirect impact has been limited and controversy exists over the risk of injury as a result of purposeful and accidental heading of the ball. Resent research on concussion in professional American football make it possible to characterize the probability of this injury based on the linear and rotational responses of head.

Methods: A total of 20 tests are conducted with a ball release velocity of $\mathrm{v}_{\mathrm{r}} \sim=14 \mathrm{~m} / \mathrm{s}(50.4 \mathrm{~km} / \mathrm{hr})$. Athletes are represented by crash test dummy head-neck system. One test is conducted with a ball of lesser mass and pressure.

Results: Using a newly developed criterion called Head Impact Power (HIP), it was found that: Higher HIP values are associated with lateral impacts, with the highest numbers occurring when the vertical axis (z-axis) of the model is perpendicular to the trajectory of the ball. Lowest HIP values occurred where the $\mathrm{z}$-axis of the head was 45 degrees to the ball trajectory. All impacts produced a less than $2 \%$ probability of concussion.

\section{NEUROSURGICAL COMPLICATIONS OF MILD HEAD INJURIES}

V. Beneš Department of Neurosurgery, 1st Faculty of Medicine, Charles University, Postgraduate Institute of Medical Studies and Central Military Hospital, Prague, Czech Republic

Objective: To summarize possible surgically treatable complications of mild head injuries. being studied throughout the world. This issue of the British fournal of Sports Medicine, which serves as the conference abstract book, also contains a number of topical articles, reviews and case reports on concussion. As the Editor of this prestigious international sports medicine journal, I would hope that delegates attending the symposium would take the time to read the Journal and appreciate our commitment to high quality evidence-based sports medicine.

The first recorded mention of concussion as a transient form of head injury was by the great Persian physician and philosopher, Rhazes, in approximately $950 \mathrm{AD}$. Over 1000 years later, we are only beginning to put the pieces of the concussion injury jigsaw in place that will enable a complete understanding of this problem. This Symposium is an important step in this journey.

DR PAUL MCCRORY Scientific Program Committee, International Symposium on Concussion in Sport

Design: Review.

Settings: major referral neurosurgical centre at Prague, Czech Republic.

Neurosurgical complications are best divided according to the time course of their development and according to the structures involved.

- Immediate complications: Symptomatology usually dominates over that of cerebral concussion. In typical cerebral concussion or mild head injury the skull may be fractured at any anatomical location. Skull convexity linear fractures are of no therapeutic consequence unlike depression fractures which should be considered for surgical treatment. Skull base fractures, not infrequently without any brain damage, are of importance due to the numerous structures involved - dural tear may result in rhinorrhea, pneumocephalus, meningitis, and cerebral abscess. Injury of the cranial nerves causes relevant neurological symptomatology. Injury of the carotid artery may cause artery thrombosis, aneurysm/ pseudoaneurysm, carotid-cavernous fistula with all the relevant consequences.

- Early complications: Symptomatology develops over the hours to days after the concussion. Apart from some of the above mentioned the most frequent and dangerous are various haematomasepidural, subdural, and intracerebral.

- Late complications: Symptomatology develops over weeks to months after the initial trauma. The most frequent is chronic subdural haematoma and rare delayed intracerebral haematoma. Apparently many of the complications caused by skull base fracture may cause delayed symptoms (pneumocephalus, carotid pseudoaneurysm etc).

Diagnostic procedures and management of the complications: Diagnostic work up and management of neurosurgical complications is the domain of neurosurgeons. Minor complications-for example, depression fractures or chronic subdural haematomas - can be treated at any institution, more complex ones-for example, complex skull base fractures with rhinorrhea or carotid injuries - should be treated 
at major institutions with access to other specialists (maxillo-facial surgeon, interventional neuroradiologist etc).

Conclusion: Neurosurgical complications of cerebral concussions are rare but dangerous and should always be kept in mind when handling the patient who has suffered a cerebral concussion.

\section{PROCEDURES AFTER MINOR TRAUMATIC BRAIN INJURY MTBI IN ICE HOCKEY TO PREVENT NEUROLOGICAL SEQUELAE}

N. Biasca, ${ }^{1}$ R. Agosti, ${ }^{2}$ Y. Tegner, ${ }^{3}$ H. Battaglia,${ }^{4}$ C. Gerber. ${ }^{5}{ }^{1}$ Rules and Technical Committees of the International Ice Hockey Federation (IIHF), Orthopedic University Hospital Zurich, Balgrist, CH-8008 Zurich, Switzerland; ${ }^{2}$ University Hospital Zurich, Zurich, Switzerland; ${ }^{3}$ Institution of Health Sciences, Lulea University of Technology, Sweden; ${ }^{4}$ Swiss Accident Insurance Company SUVA, Luzern, Switzerland; ${ }^{5}$ Orthopedic University Hospital Zurich, Balgrist, CH-8008 Zurich, Switzerland.

Over the last 15 years we are observing worldwide an alarming increase in the rate of $\mathrm{mTBI}$ in ice hockey despite improved protective gear. According to national and international statistics the proportion of mTBI to the overall number of injuries fluctuates, in ice hockey between $2 \%$ and $20 \%$, in American football between $5 \%$ and $24 \%$, and in soccer between $4 \%$ and $22 \%$. In the Canadian Hockey League the proportion of mTBI rose from $4 \%$ of all injuries in the period $1991-96$ to $8 \%$ in 1997 and to $17 \%$ in 2000 . In the professional Ice Hockey League NHL, the proportion of mTBI is increasing as well, from $2 \%$ in the season $1989-90$, to $8 \%$ in the last two seasons 1999 01. Similar increases were observed in Sweden and Switzerland. The primary neuropathology of a mTBI is a diffuse axonal injury DAI through shearing forces by sudden acceleration-deceleration. There is no universal definition of mTBI nor a standardized assessment tool. We adopted the American Academy of Neurology's 1996 suggestions. Confusion and amnesia are the hallmarks and are difficult to assess. Many of the definitions were constructed in regard to return-to-play guidelines. It has become clear that in the minutes to days following a mTBI, many brain cells remain in a vulnerable state. Long term derangements at a cellular level include calcium accumulation, elevation of lactate, decreased glucose metabolism, axonal disconnection, and neurotransmitter disturbances. The brain cells are then particularly vulnerable to additional trauma, minor changes in cerebral blood flow and/or increases in intracranial pressure, and especially anoxia. These neurometabolic changes may lead to long term physical, neurocognitive, behavioral, and emotional changes (for example, Post Concussion Syndrome). In rare cases additional mTBI and extracranial trauma may lead to cerebral edema with rapid neurological deterioration or even death (Second-Impact Syndrome). The current return-to-play guidelines do not respect this new findings appropriately - for example, allow return to the same play. The risk of further injuries is also increased. For these reasons, we recommend that any confused player with or without amnesia should be taken off the ice and not be permitted to play again for at least 24 hours.

\section{NEUROPSYCHOLOGICAL PERFORMANCE OF ATHLETES INVOLVED IN CONTACT AND NON-CONTACT SPORTS: A PRELIMINARY STUDY.}

G. Boulard, ${ }^{1}$ P. Frémont, ${ }^{2}$ G. Langlois. ${ }^{2}{ }^{1}$ Quebec Physical Deficiency Readaptation Institute, Quebec, Canada $;{ }^{2}$ Laval University Hospital Research Center, Laval University, Quebec, Canada.

Objective: To compare the neuropsychological performance of athletes involved in sports characterised by frequent mild traumatic brain injury (with or without an history of concussion) to that of athletes in non-contact sports.

Design: This is a case-control study where the independent variables are the history of concussion and/or involvement in a contact sport and the dependent variable is the neuropsychological performance

Setting: Volunteers from the university elite sport program in Quebec, Canada.

Subjects: Members of the American football team with ( $n=48$; age $21.7 \pm 1.9)$ or without $(n=50$; age $21.9 \pm 1.8)$ a history of concussion and a control group of athletes with no history of concussion nor significant involvement in a contact sport $(n=19$; age $22.2 \pm 1.9)$. All subjects were male.

Methods: Each athlete completed a questionnaire on the perceived history of concussion. The neuropsychological evaluation was performed by an evaluator blind to the perceived history of concussion and involvement in contact sport. The evaluations were performed prior to the beginning of the fall 1999 or 2000 football season and included the WAIS-III digit symbol (DSY) and digit span (DSP) tests, the Stroop Color and Word test (STR), the Brown Peterson Consonant Trigrams test (BPT), the Controlled Oral Word Association test (CWA), and the Rivermead (RIV) post concussion symptom questionnaire. For each component, the groups were compared by ANOVA and, when a significant variation was detected, Bonferroni multiple comparison test was performed.

Results: No age difference was observed between groups. Significantly better performance was observed in the control group compared to both the concussion and contact sport groups for the DSY, STR, and BPT tests as well as for the RIV symptom questionnaire (results are presented as mean and 95\% CI). No difference was observed between any group for the DSP and the CWA tests nor between the athletes involved in contact sports with and without a history of concussion for any of the tests.

Table 1 Neuropsychological performance of athletes involved in contact and non-contact sports

\begin{tabular}{|c|c|c|c|}
\hline & Concussion & Contact & Control \\
\hline DSY & $84.4(81.0-87.7)^{\dagger}$ & $83.3(79.4-87.3)^{\dagger}$ & $93.4(88.9-97.8)$ \\
\hline STR & $54.6(51.8-57.5)^{\dagger}$ & $55.6(52.7-58.5)^{\dagger}$ & $48.6(45.6-51.5)$ \\
\hline $\mathrm{BPT}^{\star}$ & $12.3(11.7-12.9)$ & $11.7(11.1-12.4)^{\dagger}$ & $13.3(12.8-13.9)$ \\
\hline RIV & $12.7(10.4-15.0)^{\text {t未 }}$ & $7.7(6.3-9.0)$ & $6.2(2.9-9.5)$ \\
\hline
\end{tabular}

"Results presented are for the 9 sec delay component but $\mathrm{p}<0.05$ for all delays. 'Different from control $(\mathrm{p}<0.05)$

${ }^{\ddagger}$ Concussion different from contact $(\mathrm{p}<0.05)$.

Discussion: The interpretation of the results from this preliminary study is limited by the relatively small number of athletes involved and by its transversal nature. Neuropsychological performance impairment might be associated to participation in contact sport as much as with the self reported history of concussion. Athletes reporting a history of concussion present significantly more symptoms than other athletes. These results further support the need for prospective studies on the effect of mild traumatic brain injuries in sports.

\section{OVERVIEW OF CONCUSSION}

\section{R.C. Cantu. Neurosurgery Service, Emerson Hospital, Concord, MA, USA}

Concussion is derived from the Latin concussus, which means "to shake violently". Initially it was thought to produce only a temporary disturbance of brain function caused by neuronal, chemical, or neuroelectrical changes without gross structural change. We now know that structural damage with the loss of brain cells does occur with some concussions. In the last several years, the neurobiology of cerebral concussion has been advanced. It has become clear that in the minutes to days following concussive brain injury, brain cells that are not irreversibly destroyed remain alive but in a vulnerable stated. These cells are particularly vulnerable to minor changes in cerebral blood flow and/or increases in intracranial pressure and especially anoxia. This vulnerability appears to be caused by an uncoupling of the demand for glucose, which is increased after injury, while cerebral blood flow is reduced. Although the precise mechanisms of this dysfunction are still in the process of being fully explained, it is now clear that although concussion in and of itself may not produce extensive neuronal damage, the surviving cells are in a state of vulnerability characterised by a metabolic dysfunction that can be thought of as a breakdown between energy demand and production. Precisely how long this period of metabolic dysfunction lasts is not yet fully understood. Unfortunately, at present, there are no neuroanatomic or physiologic measurements that can be used to precisely determine the extent of injury with concussion, nor the severity of metabolic dysfunction, nor precisely when the concussion has cleared. It is precisely this fact that makes return to play decisions after a concussion a clinical judgment.

While there is no universal agreement on the definition and grading of concussion, most tend to focus on loss or retention of consciousness (LOC) and on post traumatic amnesia as hallmarks in the grading schemes and tend to downplay the other signs and symptoms of concussion. More recent prospective studies of athletic concussion suggest all symptoms of concussion including feeling of being stunned or seeing bright lights, light headedness, vertigo, loss of balance, headaches, cognitive and memory dysfunction, tinnitus, blurred vision, difficulty concentrating, lethargy, fatigue, personality changes, inability to perform daily activities, sleep disturbance, and 
motor or sensory symptoms, must be considered. Furthermore, prospective studies have shown a poor correlation (or none) between brief periods of LOC and subsequent neuropsychological test scores raising serious doubts about any grading scale that heavily weighs brief LOC.

This paper will discuss the current role of neuropsychological testing in concussion, analyse the various grading schemes and present a revised (table 2) system based on prospective data.

Table 2 Evidence based Cantu revised concussion grading guidelines

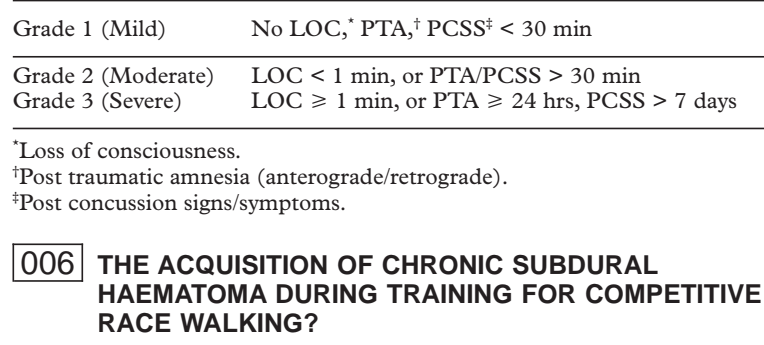

M.R. Carmont, W. Mahattanakul, T. Pigott. The Walton Centre for Neurology and Neurosurgery, Lower Lane, Fazakerley, Liverpool, UK.

Abstract: A 65 year old gentleman, anticoagulated for cardiac problems, developed a hemiparesis whilst training for race walking competitions without history of significant head injury. A computed tomography scan revealed a chronic subdural haematoma (fig 1), which was drained successfully at operation (fig 2). There has been no evidence of reaccumulation and he continues to race walk competitively.

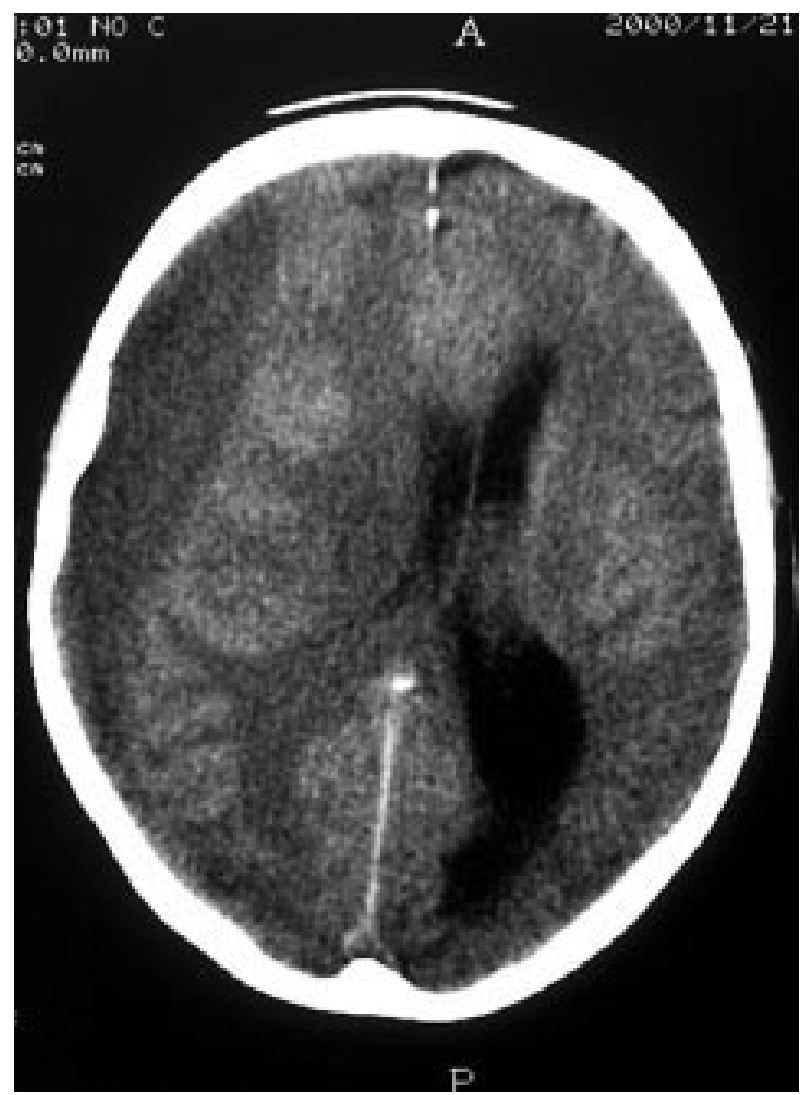

Figure 1 Preoperative computed tomography scan revealing right sided, hypodense, subdural haematoma causing midline shift and ventricular compression.

Although the causes of chronic subdural haematoma are multifactorial, we believe this is the first case in association with the jarring competitive action of race walking.

Introduction: Race walking is a physically demanding sport, commonly resulting in musculoskeletal injuries, however central nervous system insult is more rare. Competitors tend to be in good physical condition, particularly with respect to their cardiovascular

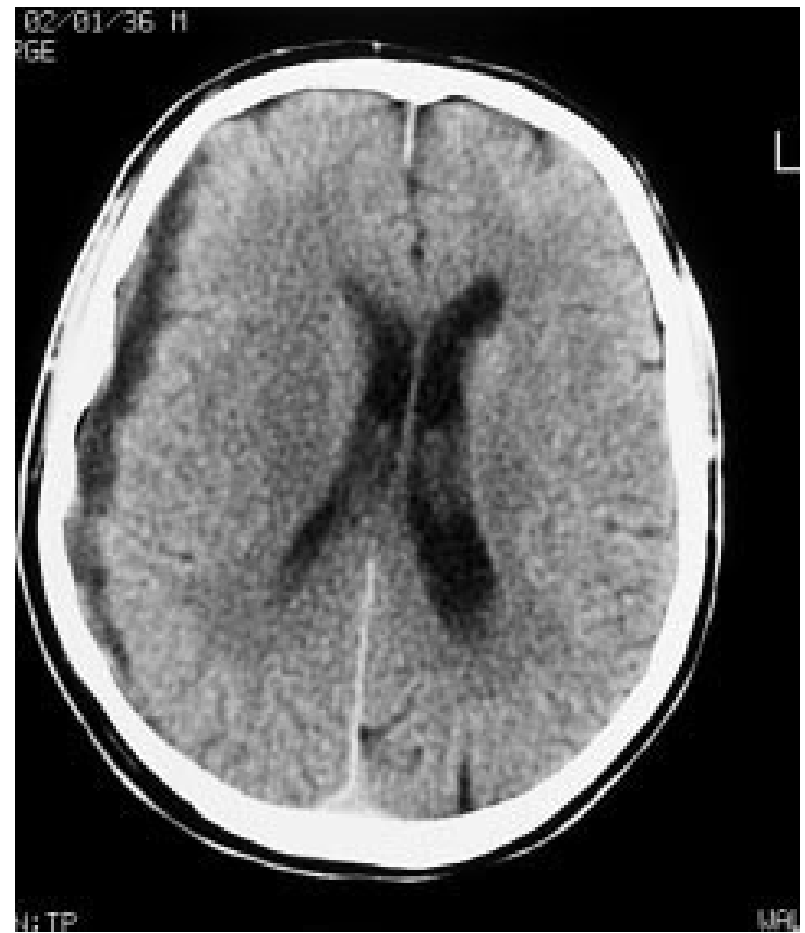

Figure 2 Postoperative computed tomography scan confirming decompression of the haematoma, re-expansion of the ventricles and near realignment of the midline structures.

systems. We present the case of a regular long distance walking competitor who suffered a chronic subdural haematoma whilst on warfarin, possibly as a result of his sporting activity.

Case Report: A brief, concise and informative description of the clinical history, physical findings, operative management, post operative progress from the onset of symptoms to the resumption of training for competitive race walking.

Discussion: A brief discussion of the aetiology and management of chronic subdural haematoma related to our patient's symptoms, physical examination findings and computed tomography images and their interpretation. Comment on the occurrence of subdural haematoma in other sports and a discussion of the rarity of chronic subdural haematoma in race walking.

The authors wish to thank the Department of Medical Photography at The University Hospital Aintree, Fazakerley, Liverpool, UK.

\section{COGNITIVE DEFICITS FOLLOWING CONCUSSION IN AUSTRALIAN RULES FOOTBALLERS}

A. Collie, ${ }^{1,2}$ P. Maruff, ${ }^{1}$ P. McCrory, ${ }^{3}$ M. Makdissi, ${ }^{3}$ D.G. Darby. ${ }^{1}$ Neuropsychology and ${ }^{2}$ Behavioural Neurology Laboratory, Mental Health Research Institute of Victoria, Australia; ${ }^{3}$ Centre for Sports Medicine Research and Education, The University of Melbourne, Australia.

Background: Cognitive impairments are a reliable consequence of mild head injury and concussion. Most prior studies of cognition following concussion in athletes have employed conventional 'paperand-pencil' neuropsychological tests. These tests often have lengthy administration times, making the acquisition of a cognitive profile a time-consuming exercise, and restricting post-concussion data collection to one or only a few cognitive domains. We administered a brief (15 min) computerised test battery assessing multiple cognitive domains to a series of concussed Australian Football League (AFL) footballers, to comprehensively characterise post-concussion cognitive impairments.

Methods: CogState ${ }^{\mathrm{TM}}$ was administered to 240 AFL footballers preseason. Fifteen footballers were assessed two and 14 days after receiving a concussive injury during the season. A group of matched non-injured footballers tested serially acted as a comparison group. All participants also completed the Digit Symbol Substitution Test (DSST) and the Trail Making Test (TMT) at each assessment.

Results: Significant impairments were evident on computerised tests of psychomotor function 2 days post-concussion. These were characterised by an increase in reaction time variability and in psychomotor 
speed. Performance in other cognitive domains remained intact. Performance was never abnormal on the DSST and TMT. All impairments had resolved at 14 days post-concussion.

Conclusions: Increases in speed and variability of psychomotor responses occur in head injured athletes. Computerised cognitive tests are a fast and accurate way of identifying such impairment. CogState ${ }^{\mathrm{Tm}}$ is a valid measure of cognitive function in sports concussion settings.

\section{TEST-RETEST RELIABILITY OF CogState ${ }^{\text {TM }}$ : A BRIEF, COMPUTERISED COGNITIVE FUNCTION TEST DESIGNED FOR SERIAL ASSESSMENT.}

D. Darby, ${ }^{1,2}$, P. Maruff ${ }^{1,3}$ A. Collie. ${ }^{1,2}{ }^{1}$ Neuropsychology and ${ }^{2}$ Behavioral Neurology Laboratory, Mental Health Research Institute of Victoria, Australia; ${ }^{3}$ Centre for Neuroscience, The University of Melbourne, Australia; School of Psychology, La Trobe University, Australia.

Background: CogState ${ }^{\mathrm{TM}}$ is a brief (15min), computerised test designed for the serial assessment of cognitive function in mildly impaired individuals. CogState ${ }^{\mathrm{TM}}$ measures a range of cognitive functions including memory, attention, problem solving, and decision making. The test-retest reliability of CogState ${ }^{\mathrm{TM}}$ was determined in a number of healthy samples, and compared to the reliability of some conventional neuropsychological tests that are commonly used to measure cognitive function in concussed athletes.

Methods: One hundred and sixty healthy individuals were assessed serially with CogState ${ }^{\mathrm{TM}}$ and with conventional neuropsychological tests. Of these individuals, 60 were aged between 18 and 30 years (young group) and 100 were aged between 50 and 85 years (older group).

Results: In both groups, test-retest reliability values for CogState ${ }^{\mathrm{TM}}$ subtests were generally higher $(0.5-0.8)$ than those recorded for conventional neuropsychological tests $(0.2-0.6)$. Further tests of reaction time (RT) displayed better reliability than tests of accuracy. In addition, reliability values were higher in the young group than in the older group.

Conclusions: Computerised cognitive tests have psychometric properties that provide excellent reliability when such tests are administered serially to healthy individuals. Such tests are therefore ideal for measuring cognitive change caused by concussion or other neurological insults.

\section{CONCUSSIONS AMONG CANADIAN UNIVERSITY FOOTBALL AND SOCCER PLAYERS}

J.S. Delaney, V.J. Lacroix, S. Leclerc, K.M. Johnston. McGill Sport Medicine Clinic, McGill University, Montreal, Quebec, Canada.

Objective: A study to examine the incidence and characteristics of concussions for one season of Canadian university football and soccer.

Design: Retrospective survey.

Participants: 328 Canadian football and 201 soccer players reporting to 1999 fall training camp.

Main Outcome Measures: Based on self-reported symptoms, calculations were made to determine the number of concussions experienced during the previous season, the duration of symptoms, the positions most commonly affected, and any associated risk factors for concussions.

Results: Of all the athletes who returned completed questionnaires, $70.4 \%$ of the football players and $62.7 \%$ of the soccer players had experienced symptoms of a concussion during the previous season. Only $23.4 \%$ of the concussed football players and $19.8 \%$ of the concussed soccer players realized they had suffered a concussion. More than one concussion was experienced by $84.6 \%$ of the concussed football players and $81.7 \%$ the concussed soccer players. When examining symptom duration, $51.6 \%$ of all concussed football players and $44.3 \%$ of all concussed soccer players experienced symptoms for at least one day or longer. Tight ends and defensive lineman were the positions most commonly affected in football, while goalies were the players most commonly affected in soccer. Variables which increased the odds of suffering a concussion for football players included a past history of a recognised concussion while playing football. Variables which increased the odds of suffering a concussion for soccer players included a past history of a recognised concussion while playing soccer and being female.

Conclusion: University football and soccer players seem to be experiencing a significant yet comparable amount of concussions while participating in their respective sports. Variables which seem to increase the odds of suffering a concussion during the previous season for football and soccer players include a history of a recognised concussion. Despite being relatively common, many players may not recognise the symptoms of a concussion.

\section{COMPARATIVE REVIEW OF USA CONSUMER PRODUCT SAFETY BOARD DATA FOR SOCCER, ICE HOCKEY, AND AMERICAN FOOTBALL FROM 1990 TO 1999}

J.S. Delaney, ${ }^{1}$ S.E. Brien, ${ }^{2}$ E. Baron.${ }^{1}{ }^{M c}$ Gill Sport Medicine Clinic, McGill University, Montreal, Quebec, Canada, ${ }^{2}$ Hotel Dieu Hospital, Windsor, Ontario, Canada.

Objective: To examine the incidence of head injuries in three popular American team sports.

Sample: Patients presenting and treated at participating hospital emergency departments in the USA.

Data: Analysis of the data from the National Electronic Injury Surveillance System (NEISS) for the USA.

Study: NEISS estimates are based on a sample of participating USA hospital emergency departments rather than a census of all USA hospital emergency departments. Calculations are based on participating hospitals' data to arrive at a national estimate for the total number of treated head injuries in USA hospital emergency departments. The data, provided by the USA Consumer Product Safety Board, was reviewed and summarised as follows; there were an estimated 86697 head injuries in soccer, 17008 in ice hockey, and 204802 in American football that presented to USA emergency departments from 1990 to 1999 . When we examined injuries in relation to participation rates per year, in 1999 the following numbers were estimated for head injuries per participant per year; soccer $0.10 \%$, hockey $0.11 \%$, and American football $0.13 \%$. Cumulative skull and brain injuries (concussions, fractures, and internal injuries) presenting to emergency departments from 1990 to 1999 were 50035 in soccer, 9883 in ice hockey, and 128968 in football. Comparing years 1990 to 1999 , head injuries have increased $150 \%$ in soccer, $134 \%$ in ice hockey, and $62 \%$ in American football. Concussion as an injury group has increased $250 \%$ in soccer, $269 \%$ in ice hockey, and $77 \%$ in football from 1990 to 1999 .

Conclusion: While American football has experienced the highest number of head injuries and concussions from 1990 to 1999 , both soccer and ice hockey also report high incidences of both head injuries and concussions. It is notable that ice hockey and soccer report dramatic increases in both head injuries and concussions. This report raises concerns regarding the risk of head injuries in soccer, football and ice hockey, especially since the NEISS data reports only those injuries which were seen and treated in hospital emergency departments across the USA.

\section{STATISTICAL TECHNIQUES FOR INTERPRETING POST-CONCUSSION NEUROPSYCHOLOGICAL TESTS}

D. Erlanger, D.J. Feldman, J.T. Barth. Columbia University, New York, USA; HeadMinder, Inc., New York, USA; Univeristy of Virginia, Charlottesville, USA.

Introduction: Neuropsychologists are frequently consulted to aid in determining whether a head injured athlete should return to play. However, there is scant clinical literature regarding how their post-concussion test results should be analysed. One proposal, based on the supposition that practice effects always result in improved scores, is to consider any decrease from baseline as evidence of impairment. Another proposal is to use the standard error of difference. Although multiple regression has been proposed as a third alternative inasmuch as it accounts for both practice effects, regression to the mean, and demographic variables, no clinical data has yet been published.

Methods: We administered two reaction time tests twice to 164 healthy athletes aged 14 to 35 . From these scores we derived normative data. We then administered baseline tests to 854 athletes, 27 of whom subsequently sustained concussions.

Results: We found significant evidence of differential effects of regression to the mean on both Simple and Complex Reaction Time tests, $(\mathrm{t}=2.53, \mathrm{p}<0.05, \mathrm{t}=2.46, \mathrm{p}<0.05$, respectively). In the normative group, low scorers at baseline had faster times at a second test administration. High scorers' performances, in contrast, slowed relative to their baselines. Athletes who sustained concussions were 
more accurately classified as impaired vs recovered using multiple regression than using other methods.

Discussion: Athletes who performed well on reaction time tests at baseline were likely to perform more slowly on a subsequent test administration regardless of whether they had sustained a concussion; this is expected due to the statistical phenomenon of regression to the mean. The statistical technique that accounts for this phenomenon is multiple regression, which was the most accurate method for classifying performances of 27 athletes who sustained concussions.

\section{A CHRONOMETRIC APPROACH TO ASSESSMENT OF MILD HEAD INJURY IN SPORT}

D. Goodman, ${ }^{1}$ D. Meichenbaum, ${ }^{1}$ M. Gaetz, ${ }^{1}$ E. Roy. ${ }^{2}{ }^{1}$ Simon Fraser University, Burnaby, Canada ${ }^{2}$ University of Waterloo, Kitchener, Canada.

Introduction: A reduction in the speed of information processing may be a common underlying factor involved in the decrement of cognitive functioning following mild head injury. This may be of particular relevance in activities such as ice hockey, in that quick response times are an essential component of play. Moreover, in players who have previously suffered a concussion, and may be still suffering the after-effects, the appropriate and quick response to unanticipated actions may be the response that prevents further injury.

Methods: In this study we examined both the acute and recovery period effects of mild head injury of hockey players on choice reaction time in both compatible and incompatible situations. This was accomplished by comparing the players post-injury scores and post-injury recovery period scores to baseline performance levels. Prior to the season's start of play, baseline data were collected from $\mathrm{n}=555$ (over two years) Canadian Junior Hockey League players. The 46 players who subsequently sustained a concussion underwent a serial battery of neuropsychological tests within 24 hours of the injury, which was repeated approximately one week later.

Results: The averaged (over the $1,2 \& 4$ choice) reaction time of players was significantly slower immediately following mild head injury (303 ms) when compared to baseline assessment $(280 \mathrm{~ms})$ measures. The reaction time subsequently decreased to near baseline levels $(269 \mathrm{~ms})$ after a one-week recovery period. As expected, the 1-choice RT $(251 \mathrm{~ms})$ was faster than the 2 -choice RT $(287 \mathrm{~ms})$, which were both faster than the 3-choice RT (332 ms).

Reaction times in the incompatible situation revealed similar results, though, as expected, the differences between conditions was somewhat larger. We further noted that an assessment of the variability in reaction times was also a sensitive indicator of performance decrements, with the standard deviation of reaction time in the compatible conditions increasing to $79 \mathrm{~ms}$ over baseline of $61 \mathrm{~ms}$, and in the incompatible conditions going from baseline of $61 \mathrm{~ms}$ to $84 \mathrm{~ms}$. In both cases the variability returned to baseline levels one week post concussion.

Conclusion: A chronometric approach seems well advised in assessment of mild head injury. This assessment, however, should include a choice reaction time paradigm, under both compatible and incompatible conditions to enhance the sensitivity of the test. In addition, measures of variability may also be important indicators of decreased cognitive functioning.

Acknowledgments: This work was supported by grants from the British Columbia Neurotrauma Initiative and the Canadian Institutes of Health Research.

\section{THE EFFECT OF IMPACT SEVERITY AND MATERIAL DENSITY ON ENERGY ATTENUATION}

T.B. Hoshizaki, ${ }^{1}$ E. Spyrou. ${ }^{2}{ }^{1}$ University of Windsor, Windsor, Ontario, Canada ${ }^{2}$ The Hockey Company, Montreal, Quebec, Canada.

Objective: To determine the ability of expanded polypropylene to attenuate energy from impacts of varying severity.

Design: We employed three densities of foam, $55 \mathrm{~kg} / \mathrm{m}^{3}, 70 \mathrm{~kg} / \mathrm{m}^{3}$, and $90 \mathrm{~kg} / \mathrm{m}^{3}$ measuring $125 \times 125 \mathrm{~mm}$ and $19 \mathrm{~mm}$ thick. A monorail drop test apparatus was used to impact the foams with two types of impactors flat and spherical. An accelerometer was used to record acceleration during the impact. Two impact energy levels was used, $20 \mathrm{~J}$ and $40 \mathrm{~J}$, repeated three times. The dependent variable was acceleration measured in gravity $(\mathrm{g})$. The research model consisted of a S4 $(\mathrm{I} 2 \times \mathrm{E} 2 \times \mathrm{D} 3) \times \mathrm{T} 3$.

Results: All three main effects were significant $(95 \%$ level of confidence). Across the foam densities, the trials and impact energy levels. Closer analysis of the data revealed impacts on the flat anvil at low energy $(20 \mathrm{~J})$ resulted in significantly higher acceleration values (g). In the case of the $20 \mathrm{~J}$ impact on the stiff foam the acceleration doubled from 90 g's to 180 g's. This difference between the two anvils was exaggerated as the foam became stiffer. When the data for the $40 \mathrm{~J}$ impact was observed across the three trials, impacts on the two lower density foam using the spherical impactor resulted in significantly higher values in the second and third impacts.

The low density foams recorded $122 \mathrm{~g}$ 's on the first trial increasing to 585 g's by the third trial when using the spherical impactor set at $40 \mathrm{~J}$. The $70 \mathrm{~kg} / \mathrm{m}^{3}$ foam increased from $110 \mathrm{~g}$ 's to $294 \mathrm{~g}$ 's from the first to third trial using the spherical impactor at $40 \mathrm{~J}$.

Conclusions: Recently ice hockey helmet designers have been attracted to employing EPP foam in helmets to address more stringent standards. Unfortunately this has generally resulted in stiffer foams. Limited history and experience in using these foams increases the unpredictability of the foams under different stresses. This study provides some insight into what is a complex interaction between EEP foam and impact severity.

\section{AIBA EXPERIENCE IN CONCUSSION}

P. Jako. Amateur International Boxing Association, Medical Commission

The modern age of boxing began at the end of the 19th century. Since then, many adverse health effects occurred in boxing, including death. Although the death rate has been low in comparison to other sports, the allegations of acute and chronic brain damage resulted in a great number of articles (more than 1200 in the 20th century). Since the introduction of safety rules and more extensive medical control for amateur boxers during the last 20 years, both acute and chronic brain damage have decreased, and amateur and professional boxing are now absolutely two different kinds of sports. The main reasons why amateur boxing doesn't seem to be any more dangerous than most other sports are the following: (1) very careful medical monitoring, (2) sophisticated neurodiagnostic tests, (3) health protective equipment, (4) new regulations. Nevertheless, mild brain trauma or concussion with a different incidence can happen in any kind of sport, in boxing as well. The quick diagnosis of concussion in boxing is easier than in other sports. During the bout, the boxer is very closely controlled by the referee and the ringside physician. If a boxer, in the opinion of the referee is unfit to continue the contest, having received hard blows to the head, the bout shall be stopped (RSCH). If a boxer is "down" and fails to resume within ten seconds, the decision is KO. The ringside physician has the right to stop the bout at any time. The decision of $\mathrm{RSCH}$ or $\mathrm{KO}$ (without unconsciousness) does not mean necessarily the diagnosis of concussion but it can be, therefore, a very thorough medical checkup of the boxer is mandatory to determine the need and nature of further medical observation and/or hospitalisation. In the case of unconsciousness, either brief/seconds/or prolonged ( $\mathrm{min}$ ), the boxer must be transported immediately to the nearest emergency department by ambulance.

If a boxer is defeated by $\mathrm{KO}$ or $\mathrm{RSCH}$, he may not take part in boxing or sparring for a period of at least four weeks afterwards. If during a period of three months a boxer is twice defeated by KO or RSCH, then the boxer may not take part in boxing/sparring for a period of three months after the second occurrence. If within 12 months a boxer suffers three KO's or RSCH he will be suspended for one year after the third incident. Before the boxer is allowed to fight after the rest periods have elapsed, he must be cleared "fit" by a neurologist. The use of computer tomography scan or magnetic resonance imaging is recommended. It is unambiguously demonstrated that after some modifications of the rules, mandatory using of head guard, decreased duration of rounds and proper refereeing improved medical control resulted in a decreasing tendency in the ratio of those bouts which ended by KO or RSCH $(11.0-0.3 \% ; 6.4-1.5 \%)$.

\section{NEW INVESTIGATIVE TOOLS IN CONCUSSION}

\section{K.M. Johnston. McGill Sport Medicine Clinic, Montreal, Canada.}

Closed head injury in contact sport is a problem of such magnitude that improvements in diagnosis and management are now urgent. In the USA, as many as 300000 concussions are identified each year in athletic activity and certainly this number underestimates the incidence as this injury is under reported. Considerable controversy exists when evaluating and grading injury severity and making return to play decisions. This controversy exists largely because objective means to measure concussive injury have not been established. The 
purpose of our research is to develop and evaluate the use of new diagnostic techniques in concussive injury in sport. Two research studies will be described involving the use of (1) Event-Related Potentials (ERP), (2) functional magnetic resonance imaging (fMRI). We have used ERP to assess cerebral activity following mild traumatic brain injuries in college athletes practicing contact sports. Concussion victims showed a striking decrease in P300 amplitude, an effect presumed to reflect alterations in attentional-cognitive processes. Moreover, the degree of impairment was strongly related to the severity of post-concussion symptoms and recovery was seen with resolution of symptoms. Our data suggest that concussions cause objectively measurable changes in the electrophysiological markers of brain activity and hence in the functions of the structures from which they originate. ERPs may thus constitute a reliable method to accurately monitor the clinical course and recovery of head injuries in athletes.

The second study involves a preliminary investigation of possible alterations in the functional cerebral metabolic patterns following concussion. Using fMRI, we compared the activation patterns and the performances of athletes who had suffered one or more concussion to a group of healthy control subjects on both verbal and visual working memory tasks. Similar tasks have been employed and proven to be sensitive to frontal lobe function. As a group, the concussed athletes showed significantly poorer performances on both verbal and visual working memory tasks compared to the control group. In addition, fMRI data revealed that the concussed subjects displayed different activation patterns than the controls while performing the working memory tasks. Within the patient group, the activations observed are generally weaker, and of different anatomical location than those observed in the control group, suggesting a change in functional anatomy after concussion.

It is important to identify individuals at high risk for persistent postconcussive symptoms since these sometime subtle deficits can create havoc in the lives of the athletes who believe themselves to be capable of returning to sports activities immediately. The results from the present studies suggest a physiological basis to persisting post-concussive symptoms. Furthermore, by combining patients' neuropsychological profile with information derived from ERPs and fMRI, we believe we possess powerful diagnostic and prognostic tools relevant to the athlete's degree of disability and readiness to return to play.

\section{RETURN TO PLAY FOLLOWING A CONCUSSION}

J.P. Kelly. Chicago Neurological Institute, Chicago, USA.

Recommendations for return to play after concussion have been concerned primarily with the prevention of catastrophic outcome related to severe brain swelling after an additional concussion occurs while still symptomatic from an earlier one. It is also important to avoid cumulative cognitive deficits and chronic post-concussion symptoms associated with multiple concussions.

The role of the health care professional in the diagnosis of concussion in sports includes providing detailed objective assessment of the athlete and offering advice regarding safe return to competition. While there is variability in the existing scales for grading the severity of concussion, there is agreement among guidelines for returning to play which recommend that the athlete be free of symptoms before resuming any activity which may include the risk of additional concussion.

In addition to the reporting of symptoms by the athlete, the health care professional can rely on various assessment measures to evaluate the athlete's recovery from concussion. These include detailed neurological examination, mental status testing, neuropsychological assessment, and neuroimaging studies. Abnormalities detected by any of these methods should preclude the athlete from returning to play. Even when results are found to be normal, incremental conditioning and practice sessions are typically necessary to avoid returning a deconditioned and unprepared athlete to sports activities.

\section{NEUROPSYCHOLOGICAL EVALUATION OF CONCUSSION: COMPARISON OF THE MCGILL “ON-FIELD” EVALUATION TO THE NHL TEST BATTERY}

S.L. Leclerc, M. Lassonde, F. Giroux, N. Gosselin, K.M. Johnston. McGill University, Montreal, Canada.
Objective: To develop a new clinically useful "on-field" exam to evaluate the concussed athlete and compare results to the National Hockey League (NHL) neuropsychological test battery currently used.

Design: Prospective study.

Setting: Varsity football and ice hockey teams at McGill University. Patients: 187 male athletes from the varsity football team $(n=144)$ and ice hockey team $(n=43)$ at McGill University from August 1998 to April 2000.

Interventions: At pre-season exam, each athlete underwent the McGill "on-field" evaluation and the NHL test battery as a baseline measurement. During the sports season, all concussions were serially evaluated with both test batteries until concussed athletes became symptom free. All concussions were graded with the McGill grading system (Sports Medicine, in press).

Main outcome measure: Baseline scoring and post concussion scoring were compared, using both test batteries. Correlation analysis between the results were conducted.

Main results: The McGill "on-field" evaluation specifically tests orientation, immediate memory, concentration and delayed recall, and uniquely emphasizes the evaluation of post concussion symptoms.

Delayed memory and reverse digit tests, as well as post concussion symptoms were highly sensitive to detection of the concussed athlete. A statistically significant correlation exists between certain tests of the McGill on field evaluation and the NHL battery.

Conclusion: Both the McGill "on-field" evaluation and the NHL test demonstrated statistical sensitivity to detect concussed athletes. Post-concussion symptoms are important to evaluate and neuropsychological testing represents one way to detect some of the post-concussion symptoms.

Acknowledgements: Research supported by the REPAR network from the Fonds de la Recherche en Santé du Québec and the American College of Surgeons (KMJ).

\section{CONCUSSION IN SKI SPORT}

Inggard Lereim. Fédération Internationale de Ski, Medil Commitee

In countries where national accident statistics are available, accidents leading to injury are a major health problem. The most severe injuries occur in traffic accidents followed by occupational accidents. Sports injuries count for between $13 \%$ to $17 \%$ of all injuries. The majority of the sports injuries are seen in team sports like soccer and handball. In countries where skiing is an important sport, $16 \%$ of all injuries are skiing injuries. The majority of the injuries seen by skiers are injuries to the limbs, followed by head injuries in alpine skiers and snowboarders, and injuries in the back and chest in cross country skiers.

The main mechanism of trauma is fall from one level to another. The most severe head injuries however, are caused by collision with fixed obstacles like trees, poles, and with other skiers. Offence against the rules and regulations of skiing in the venue is a frequently an additional factor along with lack of protective equipments. Only a minority of leisure skiers with head injuries use a helmet. In comparison competitors in downhill, giant slalom, and jumping use helmets without exception. In spite of this fact head injury may occur due to the high speeds and other high energy factors.

The percentage of head injuries in alpine skiing is $9 \%$, jumping $5 \%$, cross country $4 \%$, and snowboarding $7 \%$. Most of the head injuries are contusions and wounds, about $15 \%$ are inner head injuries like concussion. Severe head injuries count for a minority of cases and is seen in competitive alpine skiers and occasionally in jumpers. Most of the severe head injuries are seen among leisure skiers during off piste skiing. Fatal cases are also seen by collisions with fixed obstacles and machines in the venue.

In Norway a 10 year epidemiological follow up of the skiing injuries has been completed. Accident prevention and injury reducing measures based upon knowledge gained from the first year of registration are being carried out. A significant reduction of the number of injuries as well the average injury severity has been seen.

Prevention of head injuries in sport, like other injuries, must be based upon information, technical measures at the venues, change of rules and regulations, and a consequent use of personal protective equipment such as helmets. Skiers with head injuries must be treated correctly with a proper primary assessment, with initial and definitive treatment based upon the existing international guidelines for the treatment of head injuries. The patient must be followed up until complete recovery. The return to physical activity must correspond to the severity of the injury, and not to the strong desire of many athletes 
to have a very short break in their sporting activities. The professional authority of the responsible doctor must not be overruled by non-professionals in the sports organisation.

\section{NEUROPSYCHOLOGICAL TESTING IN SPORTS: PAST, PRESENT AND FUTURE}

M.R. Lovell, ${ }^{1}$ M.W. Collins,,${ }^{1}$ F.H. Fu, ${ }^{1}$ C.J. Burke, ${ }^{1}$ J.C. Maroon, ${ }^{2}$ K. Podell,${ }^{3}$ J Powell. ${ }^{1}$ Department of Orthopaedic Surgery and Sports Medicine, University of Pittsburgh Medical Center, Pittsburgh, Pennsylvania, USA; ${ }^{2}$ Department of Neurosurgery, University of Pittsburgh medical Center, Pittsburgh, Pennsylvania, USA; ${ }^{3}$ Division of Neuropsychology, Henry Ford health System, Detroit, Michigan, USA; ${ }^{4}$ Department of Kinesiology, Michigan State University, East Lansing, Michigan, USA.

Introduction: Over the past decade, neuropsychological testing has evolved into a valuable tool in the diagnosis and treatment of cerebral concussion. In the United States, neuropsychological testing is now routinely utilised by the majority of National Football League (NFL) teams and has been mandated for all athletes within the National Hockey League (NHL). The utilisation of neuropsychological testing in amateur athletes has been limited by a number of practical issues including a limited number of neuropsychologists with specific training in sports medicine and the time demands of traditional "paper and pencil" neuropsychological test instruments.

Objective: This article reviews the history of neuropsychological testing in sport with a specific focus on the NFL and NHL programs. In addition, the recent use of computerised neuropsychological test batteries in sports will be reviewed with specific reference to a programme that is currently being utilised by 60 high schools and 30 colleges within the United States (ImPACT).

Method: Preliminary data from over 100 concussed high school and college athletes is presented and is compared to and athlete control group, matched by age.

Results: The results of a Multivariate Analysis of Variance (MANOVA) analysis reveals highly significant differences between the concussed and control groups in the areas of memory, visual processing, and reaction time at 24 hours $(\mathrm{F}=6.74, \mathrm{p}<0.0005)$ and three days post-injury $(F=5.50, p<0.001)$. Significant differences between the groups were evident at five days post-injury on the memory composite index of ImPACT. No statistically significant differences were evident between the groups at seven days post-injury, although subtle differences between the concussed and control groups were evident.

Conclusions: Neuropsychological testing has become increasingly utilised in the diagnosis and treatment of concussion. The use of computerised neuropsychological test batteries holds great promise in the evaluation of the concussed athletes.

\section{COMPUTERISED COGNITIVE ASSESSMENT OF CONCUSSION IN AUSTRALIAN RULES FOOTBALL}

M. Makdissi, ${ }^{1}$ A. Collie, ${ }^{2}$ P. McCrory, ${ }^{1}$ D. Darby, ${ }^{3}$ P. Maruff, ${ }^{4}$ K. Bennell. ${ }^{1}$ ${ }^{1}$ Centre for Sports Medicine Research and Education, University of Melbourne; ${ }^{2}$ Neuropsychology Laboratory, Mental Health Research Institute of Victoria; ${ }^{3}$ Behavioral Neurology Laboratory, Mental Health Research Institute of Victoria; ${ }^{4}$ School of Psychological Science, La Trobe University.

Objective: Neuropsychological tests play an important role in the management of sports related concussions. They provide objective information on the athlete's cognitive function and thus facilitate decisions on safe return to sport. Computerised neuropsychological tests theoretically offer a number of advantages over the tests currently being used in the management of sports related head injuries, however their role in this domain is yet to be established. The purpose of this paper is to present a case series of concussions observed in Australian Rules football. These are part of a prospective cohort study being conducted in 240 Australian Rules footballers, the aim of which is to investigate the role of computerised cognitive tests in the assessment and follow up of sports related concussions.

Methods: Baseline measures on the Digit Symbol Substitution Test (DSST), Trail Making Test-part B (TMT) and a computerised cognitive test battery (Cogstate) were obtained on 240 players. Tests were repeated in players who had sustained a concussive injury. A group of non-injured players were used as matched controls. For the computerised test battery, only data from tests of Simple Reaction Time (SRT) are reported.

Results: A total of six concussions were observed over a period of nine weeks. At follow up, DSST and TMT scores did not significantly differ from baseline scores in both the control and concussed groups.
However analysis of the SRT data showed that concussed athletes recorded an increase in response variability from baseline to follow up. In contrast, the response variability decreased on follow up testing of control players $(\mathrm{p}<0.02)$

Conclusion: Increased variability in response time may be an important cognitive deficit following concussion. This has implications for consistency of an athlete's performance following injury, as well as for tests used in clinical assessment and follow up of head injuries in sport.

\section{QUANTIFYING THE COGNITIVE IMPAIRMENT ASSOCIATED WITH CONCUSSION: USING BLOOD ALCOHOL CONCENTRATION AS A REFERENCE POINT}

P. Maruff, ${ }^{1,2}$ A. Collie, ${ }^{1,3}$ D.G. Darby. ${ }^{2,3}{ }^{1}$ Neuropsychology and ${ }^{2}$ Behavioural Neurology Laboratory, Mental Health Research Institute of Victoria, Australia; ${ }^{3}$ Centre for Neuroscience, The University of Melbourne, Australia.

Background: The inference that mild changes in cognitive performance occur following concussion, and reflect a subtle but reversible brain injury, is well accepted in sports medicine. However, the measures used to define and communicate the magnitude of this cognitive impairment are arbitrary and generally meaningless to sportspeople. We compared directly the magnitude of the cognitive impairment following concussion to that occurring in alcohol (Blood Alcohol Concentration (BAC) $0.10 \%$ ) and fatigue (awake for 24 hours) affected individuals.

Methods: A brief (15min) computerised test battery assessing multiple cognitive domains (CogState ${ }^{\mathrm{TM}}$ ) was administered to 15 Australian Football League (AFL) footballers post-concussion, 40 healthy age matched controls with BAC of $0.10 \%$, and to the same control subjects over 24 hours of sustained wakefulness. The magnitude of cognitive impairments were determined by comparing postconcussion, alcohol, or fatigue results to baseline data recorded at earlier assessments.

Results: Impairments detected 2-3 days following concussion were of equivalent magnitude to those detected with a BAC of $0.10 \%$, and a period of sustained wakefulness of 24 hours. However, there were important differences in the pattern of cognitive impairments observed in each experimental condition.

Discussion: Concussed AFL footballers demonstrated performance impairments of equivalent magnitude to those observed in individuals with BACs twice the legal Australian driving limit. The severity of cognitive impairment in the 2-3 days following concussion are easily communicated using BAC, or hours of sustained wakefulness, as a reference point.

\section{SOCCER RELATED CONCUSSIONS:}

E.J.T. Matser. Neuroscience Centre, Erasmus University Rotterdam, The Netherlands.

Concussions in soccer, which soccer players may refer to as "dings", "having your bell rung", and "seeing stars" have received increasing attention due to concerns about longer term effects. Most concussions cause a temporary disruption in mental functioning and most concussion symptoms seen in amateur and professional soccer players fade within a week or two. In studying seven professional premier league soccer teams in The Netherlands two out of 11 players incurred grade 3 concussions during a single season (season 1999-00). Most of the concussed players were forward and defense players and head to head collisions were the main cause of concussion, another common cause of concussion was head to body contact (players running into each other, players kicked to the head) and some players were concussed by balls to the head delivered from free kicks. Although most players seem to recover quickly from concussion, 33\% of the amateur soccer players who sustained a soccer related concussion still encounter chronic cognitive problems. ${ }^{1}$

In studying 53 elite professional soccer players, ${ }^{2} 54 \%$ experienced one or more grade 3 concussions during their professional careers. In addition, $79 \%$ percent of the players reported head to head collisions which could be classified as grade 1 and grade 2 concussions (mental alterations with no LOC). Moreover, the median of the number of soccer matches played annually was 50 (range 25 to 70 ) and the median of the number of practices per week was six (range 4 to 9). Compared to control objects (elite middle distance runners and swimmers) the professionals performed poorer on verbal and visual memory, planning, and visuoperceptual tasks. The differences 
remained significant after adjustments for confounding variables (the number of concussions not related to soccer, alcohol consumption, level of education, and the number of general anaesthesias). These psychometric test scores also remained significant after a Bonferroni correction. An increasing number of grade 3 concussions incurred during soccer participation associated negatively with memory, visuoperceptual, and planning capacity. Field position also influenced performance on neuropsychological testing. Forward and defensive players performed significantly poorer on visuoperceptual and verbaland visual memory tasks. Forward and defensive players experienced a higher frequency of soccer related concussion.

In studying 33 amateur soccer players, $27 \%$ incurred one grade 3 concussion and $33 \%$ reported two to five grade 3 concussions in their soccer careers. Compared to control objects (middle distance runners and swimmers) amateur soccer players exhibited impairments in planning and memory. These scores remained statistically after Bonferroni correction and after adjustments for confounding variables (concussions unrelated to soccer, alcohol intake, level of education, and number of general anaesthesias). Concussions incurred in soccer were inversely correlated with performances on planning, attention and visual and verbal memory tasks.

1 Matser JT, Kessels AGH, Lezak MD, et al Neuropsychological impairment in amateur soccer players. ҰAMA 1999;282:971-3.

2 Matser JT, Kessels AGH, Lezak MD, et al. Chronic traumatic brain injury in professional soccer players. Neurology 1998;51:791-6.

\section{DEFINITION \& PATHOPHYSIOLOGY OF CONCUSSION}

P. McCrory. Centre for Sports Medicine Research $\mathcal{E}$ Brain Research Institute, University of Melbourne, Australia.

Concussion is a well recognised clinical entity however, its pathophysiological basis remains a mystery. A central unresolved issue is whether concussion is associated with structural changes such as occur with severe traumatic brain injury, or whether the mechanism is entirely due to reversible functional changes. This issue is clouded not only by the lack of critical data, but by confusion in terminology. The historical context of this injury refers to a transient disturbance of neurological function caused by "shaking" of the brain that accompanies low velocity brain injuries. Following pioneering experimental work, the term acceleration concussion was proposed as the generic descriptor that should be applied to all forms of traumatic brain injury. A variation on this view holds that concussion refers to the mechanism of injury and motion of the brain within the skull rather than any clinical symptoms or pathology. In an attempt to resolve this confusion, the Congress of Neurological Surgeons (CNS) proposed a "consensus" definition of concussion that has now become the accepted definition by most researchers in this field. The CNS definition states that concussion is "a clinical syndrome characterised by the immediate and transient post-traumatic impairment of neural function such as alteration of consciousness, disturbance of vision or equilibrium due to mechanical forces." This definition has a number of limitations and these will be discussed in the talk.

The pathophysiology of concussion remains contentious. Recent experimental evidence suggests that the pathogenesis of axonal dysfunction resulting from head trauma is complex. Alteration in axolemmal membrane permeability induced by impact may cause alterations in ionic flux and exert either direct or indirect effects upon the axonal cytoskeleton. In some cases this may cause structural injury although this is not a necessary accompaniment of "concussion". In addition, a cascade of neurochemical, ionic, and metabolic changes occur following experimental brain injury resulting in a mismatch of cerebral blood flow and metabolism. Each element of the cascade has a different time window that may have important implications in both assessing and treating concussed individuals.

\section{BIOMECHANICAL ANALYSIS OF CONCUSSION}

P. McCrory. Centre for Sports Medicine Research E Brain Research Institute, University of Melbourne, Australia.

In 1974, Ommaya developed the centripetal theory of cerebral 'concussion' (that is, acceleration concussion). This theory invoked the geometric structural and material properties of the cranium and its contents. In this theory, the diffuse effects of the rotational component of inertial loading are produced by a centripetal progression of strains from the outer surfaces to the core of the brain (coinciding with the midbrain and basal diencephalon). At low levels of inertial loading, injurious levels of shear strain would not extend deeper than the cortex, while strains large enough to reach the well-protected mesencephalic part of the brainstem would result in loss of consciousness. Although this model reflects the clinical aspects of moderate to severe brain injury it does not explain the clinical features of mild brain injury or concussion in the historical sense. Our published observation that brainstem phenomena can occur in the absence of significant "cortical" symptomatology and in one anecdotal case, convulsive movements occurred with the preservation of consciousness, suggest that the clinical symptomatology may be more complex that the previously held view. Recent published studies of video analysis of human concussive impact demonstrates that the range of direct impact forces causing a sport-related concussive injury were as follows: $\Delta \mathrm{v}$ mean $4 \mathrm{~m} / \mathrm{s}$ (SD 2), Head Impact Energy mean $56 \mathrm{~J}$ (SD 36), Impulse mean $29 \mathrm{kgm} / \mathrm{s}$ (SD 11). These results are in keeping with published work from motor vehicle injury studies and bicycle injuries. More importantly, a linear relationship was noted between impact forces and measures of concussive injury severity. The understanding of impact force in the human situation underpins the design and material requirements of head protectors and helmets and is a critical element in the development of helmet testing standards. These studies mean that the original centripetal hypothesis of concussion needs to be reconsidered in light of the recent research.

\section{GRADING OF SPORT-RELATED CONCUSSION}

P. McCrory. Centre for Sports Medicine Research E Brain Research Institute, University of Melbourne, Australia.

The classification of severity of concussive injury is another contentious area. More than $90 \%$ of all concussions sustained in sport are considered to be "mild" characterised by no loss of consciousness, transient confusion, and/or a brief duration of post-traumatic amnesia. The classification of concussion severity is critical for effective management. At the present time, there are at least 30 different published injury-grading systems. Most of these anecdotal scales are impractical for clinical use in sport. To date there have been no prospectively validated studies of any of the current grading systems in sport related head injury. An abundance of published grading scales is therefore available for use by physicians, athletic trainers, coaches and athletes. It is possible for injured athletes to "shop around" for a scale that suits their competitive needs rather than best medical practice. Because the different published grading scales represent the personal view of "experts" in the area rather than a consensus of scientific evidence, the final management decision is a clinical judgment in every case. The published sport related concussion severity scales can be broken down into a number of broad groupings - surrogate head injury scales, neurosurgical scales, sport specific scales, sporting injury scales, and unclassifiable scales. These will be discussed in more detail. In summary, no scale exists which satisfies the needs of the clinician to be both scientifically valid and practical. It is for this reason that the final diagnostic decision following a concussion is a clinical judgment in every case, and it may be entirely appropriate to deviate from a particular set of guidelines depending on the circumstances surrounding the injury.

\section{EQUIPMENT TESTING AND CONCUSSION.}

A.S. McIntosh. School of Safety Science, The University of New South Wales, Australia

The performance of protective headgear and helmets is determined largely by equipment testing. Such tests are governed by national standards - for example, ASTM and CSA, and international standards - for example, ISO. Standards oriented equipment tests are complemented by field and laboratory based research in which equipment is evaluated in more realistic test scenarios or via epidemiological surveys.

The two most critical properties of helmets are impact energy attenuation and load distribution. These properties reduce the magnitude of the forces applied to the head reducing the stresses and strains in the skull and brain. Through this mechanism concussion and more serious head injury may be prevented. Impact energy attenuation is assessed by dropping a headform plus helmet with a defined kinetic energy onto an anvil and measuring the acceleration of the headform. The parameters that determine the test outcome are the impact energy (drop mass and drop height), headform type (rigid or deformable), anvil (rigid or deformable) and the helmet itself. During the impact the deformation of the helmet, and test assembly 
(headform and anvil), and the helmet's ability to harness the foam liner through load distribution, determine the impact force and headform acceleration. As Work equals Force by Deformation (W= $=\int \mathrm{Fd}$ ), the greater the deformation the lower the force and acceleration.

For example the three main ice hockey helmet standards, ISO 10256, CAN/CSA Z262.1, and ASTM F-1045, require a drop test with a pass/fail criterion of $275-300 \mathrm{~g}$ headform acceleration. However, the ISO and Canadian standards utilise a rigid anvil and headform, while ASTM, also referred to in HECC, employs a deformable anvil. Helmets tested on the deformable anvil will tend to produce lower peak accelerations compared to those tested on a rigid anvil with the same energy. Also, research shows that mild head injury is probable at head accelerations in the $150-200 \mathrm{~g}$ range. Therefore, the assessment criterion may not drive helmet improvement and a greater potential reduction in concussion.

Through an examination of the impact characteristics in sportsfor example, impact energy, location, and frequency - and their relationships to concussion, it is possible to define the ideal performance properties for sports specific helmets and translate these into equipment tests. To this end match video and injury surveillance methods are an invaluable adjunct.

\section{NHL CONCUSSION PROGRAM}

W. Meeuwisse, ${ }^{1}$ C. Burke. ${ }^{2}{ }^{1}$ University of Calgary Sport Medicine Centre, Calgary, Alberta, Canada; ${ }^{2}$ University of Pittsburgh Medical Centre, Pittsburgh, Pennsylvania, USA.

For the past four years, the National Hockey League, through the NHL Team Physicians Society Injury Committee has been conducting a project to prospectively study mild traumatic brain injury (MTBI). This program started with the preseason baseline neuropsychological (NPT) testing of all NHL players. Team physicians complete an initial evaluation form if any head injury occurs, and repeat NPT testing is conducted. A formal physician follow-up is done before players return to play. In addition, team athletic trainers report MTBI episodes as part of their ongoing injury surveillance. Lastly, an analysis of injury video clips is completed to assess the mechanism of injury and the location of occurrence on the ice surface. The number of MTBI episodes has increased over the past four years, which may be attributed to differences in occurrence or changes in the reporting threshold (that is, higher sensitivity of reporting) by players, trainers, and physicians. Variables under study include injury mechanism, initial symptom scoring, NPT changes, equipment (helmet, mouthguard use), environmental factors (boards/glass), and return to play timelines. The greatest benefit of the program to date is the increased awareness of MTBI by players and medical staff. The education regarding the significance of head injury symptoms and the discussions that have ensued regarding return to play decisions have already produced improved the detection and management of MTBI in the National Hockey League.

\section{EARLY BIOCHEMICAL MARKERS OF BRAIN INJURIES.}

T. Mussack, ${ }^{1}$ T. Graf-Baumann, ${ }^{2}$ J. Dvorak, ${ }^{2}$ M. Jochum. ${ }^{3}{ }^{1}$ Departments of Surgery and ${ }^{3}$ Clinical Biochemistry, Klinikum of the University of Munich, Germany; ${ }^{2}$ FIFA Medical Assessment and Research Center (F-MARC), Zurich, Switzerland.

Objective: To evaluate S-100B serum levels in young amateur soccer players after controlled heading for indication of early cellular brain damage.

Design: Prospective controlled cohort study.

Study group: 61 amateur soccer players (B-youth, FC Emmendingen, Germany) after controlled heading, median age 15.0 y (IQR 14.0-16.0); Stratification in three age cohorts: 11-13 y (group 1; $\mathrm{n}=7$ ), 14-15 y (group $2 ; \mathrm{n}=34$ ) and $16-18$ y (group $3 ; \mathrm{n}=20$ ). Control groups: 57 amateur handball players after training, median age 15.0 y (IQR 13.0-16.8). One hundred and five consecutively admitted minor head injured (MHI) patients (GCS score 13-15, loss of consciousness, short term memory deficits, nausea, vomiting, vertigo, seizure), median age 36.0 y (IQR 28.0-60.1).

Intervention: Venous blood samples were drawn before training, immediately and six hours after training. The neurological examination recorded the clinical status. S-100B serum levels were determined by an immunoluminescence assay (LIA-mat Sangtec 100; Byk-Sangtec, Germany). In the MHI group CCT pathologies were identified as CCT + , no CCT findings as CCT-.
Main results: No significant differences of S-100B serum levels were measured for the three age cohorts of the study group. But median S-100B levels were significantly higher in group $1(0.22 v 0.22 v 0.23)$ and group $2(0.18 v 0.24 v 0.20)$ than in group $3(0.07 v 0.09 v 0.06)$ before heading, immediately and six hours after heading. These values were not significantly different to those of the stratified handball players. At no time, the three age cohort groups reached the median S-100B levels of the CCT + group $(0.52 \mathrm{ng} / \mathrm{ml})$ being significantly elevated compared to those of the CCT- group $(0.13 \mathrm{ng} / \mathrm{ml})$.

Conclusions: Controlled heading in young amateur soccer players does not seem to influence S-100B serum release indicating early cellular brain damage. But S-100B levels were significantly higher in soccer as well as in handball players with 11-13 y and 14-15 y than with $16-18 \mathrm{y}$ of age.

\section{MOUTHGUARDS AND CONCUSSION PREVENTION}

P.M. Piccininni. York University, Toronto, Canada.

Introduction: Orofacial injuries are an unfortunate sequelae of many contact sports. In some sports, these injuries may represent up to $50 \%$ of all of the reported injuries. Mouthguards, alone or in conjunction with additional face protection, have been demonstrated by numerous studies to be an effective means of preventing or reducing dental and orofacial injuries.

Types of Mouthguards: Four main types of mouthguards are currently available. Both the stock (no customisation possible) and "boil-and-bite" varieties offer significant compromises in either or all of comfort, retention, speech, breathing, and protection and have little place in modern mouthguard discussions. In fact, some of these guards may promote concussion by compressing the TMJ complex. The vacuum-formed guard is fabricated by using suction forces to adapt heated EVA or similar material over a plaster model of the teeth. While providing reasonable fit and protection, these devices are now being replaced by guards fabricated via pressure lamination. By using both heat and pressure, serial layers of material can be tightly adapted to a plaster model to develop a guard that can be sport and athlete specific with a high degree of comfort and protection.

Mouthguards and Concussion Reduction: Numerous anecdotal reports have indicated a possible correlation between mouthguard use and concussion reduction. These reports have focussed on two primary mechanisms. The first is a dissipation of forces delivered to the maxilla, skull and TMJ complex when a blow is received by the mandible. The second is a stabilization of the skull through increased neck muscle activity when clenching, which may be enhanced with the presence of a mouthguard. This stabilization may reduce the rotation suffered by the skull when receiving an acceleration/deceleration force.

Current and Future Research: Currently, studies are concentrating on data collection and scientific reporting in professional and recreational athletes; new materials which may offer increased force dissipation without added thickness; the effectiveness of clenching in head stabilization; and determination of the optimal mouthguard design to provide protection in conjunction with an ideal separation of the condylar head and glenoid fossa. Other researchers are investigating the use of computer generated trauma models to help in quantifying some of these issues.

Conclusion: At this point mouthguards can and should be promoted as effective devices for prevention of dental and orofacial injuries. Properly designed mouthguards may also play a role in the reduction of incidence or severity of concussion, and should be considered for all athletes in contact sports, and especially those at higher risk or returning to play following a concussion.

\section{PET, SPECT AND THEIR RELIABILITY FOR DIAGNOSIS OF CONCUSSION}

B. Radanov. Wilhelm Schulthess Hospital, Zürich, Switzerland.

Sporting activities can cause brain injury as a result of either direct head impact or non-contact head trauma in connection with acceleration-deceleration. In both cases, individuals may initially present with no loss of consciousness or with superficial loss of consciousness of a short duration. These individuals are considered to have suffered mild traumatic brain injury, which does not show positive neurological or neuroimaging findings. Mild traumatic brain injury may cause numerous somatic, psychological, or cognitive complaints, the latter comprising mainly problems with attention (for 
example, complex attentional functioning) or memory. The notion that these concussion related cognitive complaints may result from organic brain damage remains to be proven by the demonstration of significant associations between the results of morpho-functional analyses (for example, PET or SPECT) and of cognitive testing. This therefore requires a reassessment of mechanisms that may contribute to traumatic brain damage and of factors that may be responsible for impaired cognitive functioning. Experimental animal studies have shown that the likelihood of traumatic brain damage depends mainly on the magnitude of acceleration-deceleration and rotation forces. However, there is no linear relationship between these forces and the extent of brain damage. In experimental research and in post-mortem studies of individuals having previously suffered mild traumatic brain injury, morphological lesions were primarily found in the periphery of the rostral brain areas. However, SPECT studies of patients after mild traumatic brain injury (loss of consciousness $<5 \mathrm{~min}$ ) demonstrated hypoperfusion in the frontal brain areas. This is of interest because the frontal brain is the site where attention is processed. More interestingly, frontal hypoperfusion has been shown to improve on follow-up investigations. In examining the relationship between SPECT and neuropsychological findings, only a weak statistical correlation is found, which is also inconsistent-that is, found only for some aspects of attentional functioning.

These findings seriously challenge the brain damage hypothesis as the cause of cognitive impairment following mild traumatic brain injury and demand alternative explanations, particularly in individuals suspected of cognitive problems that are presumed to have resulted from their involvement in sport.

Previous studies that have suggested possible brain damage in sportsmen (for example, in soccer) demonstrate a number of limitations, such as: cross-sectional in nature; unable to establish the individual's pretraumatic cognitive performance; testing restricted to neurophysiological assessments, whilst neglecting additional factors (for example, temperament) that may contribute to reduced cognitive performance in comparison with controls. Temperament, which is to a large extent inherited, plays an essential role in human executive functioning and is important for cognitive performance, emotional functioning and behaviour. Indeed, temperament may be an essential factor influencing the individual's choice of sport and may accordingly be responsible for the differences in cognitive performance observed in different sportsmen (for example, amateur soccer players $v$ swimmers).

Future research on brain damage following concussion in sportsmen should include multidimensional prospective, with appropriate sample sizes, in which the many factors that potentially influence cognitive functioning are also addressed studies (for example, PET or SPECT, psychosocial disposition and cognitive assessment).

\section{FROM HEAD CONCUSSION TO FUNCTIONAL AUTONOMY AND MAXIMISED PERFORMANCE. A DRUGLESS NEUROPHYSIOLOGICAL APPROACH TO CENTRAL SENSORY-MOTOR \& COGNITIVE RESTORATION}

P.A. Souvestre, R.O. Rothe. Neurokinetics Health Services (BC) Inc, Vancouver, BC, Canada.

Objective: To identify, correct, and prevent resisting post-concussion sensory-motor dysfunction and subsequent clinical conditions. Acute and chronic resisting conditions recovery patterns are compared and discussed.

Design: Combined Rehabilitation and Performance Restoration Programme.

Setting: Referral-based outpatient Traumatology, Sports, and Occupational Medicine Clinic.

Patients: A series of active or retired professional athletes having experienced at least one severe objective-witnessed head concussion with loss of consciousness. Some of them have been recognised as "plateaued" over several years. Will include a case review of an NHL Professional Player who comes back on the ice to play successfully in the playoffs after having experienced three major Head Concussions within the last four years. In his early 20 's, this athlete could not function well anymore in the last three years of this same time frame, and his career was decidedly at stake.

Intervention: Before starting the treatment programme, patients undergo a thorough Preliminary Evaluation (PE) series of interviews and tests, to relate the underlying neurophysiological causes to the current clinical condition, make a determination of treatability, and set the proper treatment strategy (Sensory-motor Skills Rehabilitation
Programme SSRP). Treatment consists of a drugless, non-invasive series of specific central sensory-motor stimulations, which "jumpstart" the trauma induced dysfunctional CNS areas to be restored, optimised, and maximised when possible.

Main outcome measures: Progress is tracked by subjective devices (patient keeps a daily journal of pain, symptoms, and performance factors on a 0-10 scale), and objective measurements (postural, visual, balance, and cognitive testing) which are initially performed weekly, then as needed towards end of a six month program. Evaluation is performed in a specific setting using a unique technology (DOVMS) which electronically tracks postural-visual performance in a comparable way. Symptoms are corrected within 12 weeks of the treatment programme commencing, and then performance then begins to improve.

Main results: Of nine patients who had head concussions, eight had post-concussion chronic resisting conditions (CRC). Each of the nine showed similar progression of symptom patterns following the concussions, even though the concussions occurred in different types of sports activities. These were tracked by the measurements and procedures of the NeuroKinetics Preliminary Evaluation (PE). Though they had stabilized in recovery using standard treatment modalities, after the start of treatment, all showed signs of significant improvement in pain, muscle stiffness, sensory-motor, and cognitive performance between 2-12 weeks. Medications which may have been in use by the patients to deal with these symptoms were able to be stopped.

Conclusion: After proper identification of the sensory-motor control dysfunction, our 12 week treatment protocol has been proven superior in respect to recovery pattern consistency. Recognised as a very successful "cutting edge" medical procedure since the mid- $80 \mathrm{~s}$ in Europe to treat post-concussion syndromes and related chronic resisting conditions, this innovative rehabilitation strategy and the unique related technology permitting predictable post-treatment outcomes have been awarded grants by National Research Council of Canada in 1999 and 2000.

Several case histories including post-MVA patients, NHL, and CFL professional athletes demonstrate the effectiveness of this approach that relies on:

- Specific neurophysiological paradigms

- Noninvasive technology for assessment and drugless treatment

- Comprehensive treatment and skills restoration programmes.

Over the last 16 years, many other types of non-sports patients have also benefited from this programme.

\section{CONCUSSION EXPERIENCE: SWEDISH ELITE ICE HOCKEY LEAGUE.}

Y. Tegner. The Ermine Clinic Winternet, Institution of Health Sciences, Timmermansgatan 60 University of Technology, Boden, Sweden.

The awareness of concussion in ice hockey has increased over the last two decades. In the beginning of the 80's very few physicians engaged in ice hockey were aware of the seriousness of this injury. Concussion was then considered a minor injury. In 1985 a Swedish symposium was arranged in order to classify concussion and to establish norms for treatment of this injury. At these symposia it was suggested that the term concussion should be abandoned and the term mild head injury should be used instead. Unfortunately Swedish sport physicians did not follow these recommendations.

In 1988 a survey in all of Swedish elite ice hockey was performed and $22 \%$ of the players that answered the questionnaire reported that they had a concussion. In a prospective four year study in the Swedish elite league it was found that $6 \%$ of all injuries were concussions.

Since 1984 the author has registered all injuries occurring in one of Sweden's elite ice hockey team. The injuries were registered on a special form, and for the last three years a computerised version has been used. This new computerised version allows more accurate establishing of the risk for sustaining different types of injury. Since 1987/88 the definition of a concussion used has been the same transient confusional period with or without amnesia/loss of consciousness. The classifications into different grades and treatments have varied slightly over the years. Since 1997/98 the management of concussion has, with a minor modification, followed the recommendations by American Academy of Neurology.

The Swedish elite league consists of 12 teams and the team that has been followed played between 40 and 70 league games per season.

During these 17 seasons (1984/85-2000/01) 705 injuries were registered and of these 54 were concussions $(8 \%)$. There was a great variability of the number of concussions over the years. There is 
though a clear tendency of an increase in the number of concussions per year. The first three years, when loss of consciousness was used as a definition of concussion, only one concussion was registered. The following five years a more accurate definition of concussion was used, and 10 concussions were registered. During the final five years of the registration a total of 32 concussions were found. Thus there has been a significant increase during the last years. The total number of injuries increased as well as the number of concussions, but the increase of concussions was more significant. During the first five years of the new definition $5 \%$ of the injuries were concussions and for the last 5 years $14 \%$. Most of these concussions were minor (grade 1) and grade 3 concussions with prolonged loss of consciousness were rare. One player that had sustained over 12 concussions stopped playing ice hockey due to those injuries.

This increase in concussions is alarming. Today's ice hockey is faster and more physical. This probably contributes to the high number of head injuries. There is a need for further research in order to find the accurate way to prevent and treat these injuries. 


\section{ORIGINAL ARTICLE}

\section{The trunk muscles of elite oarsmen}

\section{A H McGregor, L Anderton, W M W Gedroyc}

See end of article for authors' affiliations ......................

Correspondence to: Dr McGregor, Department of Musculoskeletal Surgery, Imperial College School of Medicine, Charing Cross Hospital, London W6 8RF, UK; a.mcgregor@ic.ac.uk

Accepted

8 February 2002
Objectives: To investigate the trunk strength of elite rowers and the impact of low back pain on these measures in order to determine if asymmetries or weakness were present.

Methods: Twenty two elite rowers were recruited: 13 reported previous low back pain, five current low back pain, and the remainder had no history of low back pain. All subjects were scanned during simulated rowing in an interventional open magnetic resonance imaging scanner. In each simulated rowing position, axial scans were obtained at the level of the L4-5 and L5-S1 disc interspace to determine the cross sectional area of the posterior trunk muscles.

Results: Considerable differences were observed between the three groups of rowers. In contrast with expectations and previous literature, the trunk muscles of rowers with low back pain had significantly larger cross sectional areas $(p<0.001)$. No left/right asymmetries were observed and no differences between oarside and non-oarside in terms of muscle cross sectional area.

Conclusion: These findings suggest that low back pain in rowers does not arise as a result of muscle weakness.

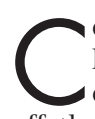
ompetitive rowing is a strenuous sport which requires high levels of dedication. It is an endurance sport associated with long hours of intensive training both on and off the water. Roy et al suggested that people with fatigue resistant back muscles and general physical fitness have fewer back problems, but the most common injury in rowers is low back pain..$^{2-6}$ There is concern that the incidence of low back pain is rising, particularly in club rowers; however, further work is required to substantiate this. Whether or not it is increasing in incidence, low back pain is a considerable problem in the rowing world. It is unclear why such injuries occur, although the many speculations include poor rowing technique and weight training skills, inadequate stretching and flexibility, and changes in the equipment. ${ }^{6-10}$

In people with low back pain, the role of the paraspinal muscles with respect to stability and functional movement has been stressed..$^{11-14}$ Particular emphasis has been placed on the multifidus muscle, the larger and most medial of the lumbar back muscles. Isolated unilateral wasting of this muscle has been observed in patients with low back pain. ${ }^{11}$ Since this initial work, it has been shown that recovery of this muscle is not spontaneous after remission of symptoms, ${ }^{15}$ and this may therefore be a factor in re-injury. However, the role of reduced muscle strength as a causative or predisposing factor in back disorders is controversial. ${ }^{16}$

Little is known about the strength of the back muscles in rowers; a pilot study investigating global parameters of strength did note that rowers did not have stronger backs than control subjects, although they exhibited greater strength in the thigh muscles. ${ }^{17}$ The significance of this finding with respect to back pain is not clear.

Rowing is an asymmetric activity which involves loading the back in a rotated and flexed position, factors already identified in back pain. ${ }^{11}{ }^{16}{ }^{18-22}$ Repetition of an asymmetric activity can lead to the development of muscle asymmetry and injury, if not addressed by appropriate training methods. Hides $\mathrm{et}_{\mathrm{al}} \mathrm{l}^{11}$ noted right to left differences in terms of muscle cross sectional area in subjects with and without low back pain, suggesting that muscle asymmetry may be important in the development of low back pain in the general population. Parkin et $a^{17}$ noted a left/right asymmetry in muscle activity during isometric contraction of the back extensor muscles.

As well as bilateral asymmetry, imbalances can occur between the agonist and antagonist muscles. Motion studies have noted changes in the motion of the pelvis during rowing in rowers with low back pain, ${ }^{23}$ which may be caused by an imbalance of back flexors and extensors and the muscles acting at the pelvis. However, relatively few studies have investigated the relation between imbalance in muscle strength and the occurrence of injuries. Previous studies have investigated muscle weakness and imbalance after injury and surgery and suggested that imbalance is associated with injury and recurrence of injuries. ${ }^{24-26}$

We examined measures of cross sectional area of the muscles acting directly on the lumbar spine (the multifidus, erector spinae, and iliopsoas) during simulated rowing in elite oarsmen with and without low back pain.

\section{METHODS}

\section{Study population}

Twenty two elite rowers ranging from international under 23 to senior I open oarsmen were recruited primarily from the Imperial College Boat Club. The mean (SD) age of the subjects was 22.6 (4.3) years. Ten subjects rowed stroke side and 12 bow side. All had been rowing for four years or more. Thirteen subjects (mean age 23.2 (5.3) years, mean weight 87.8 (8.5) $\mathrm{kg}$ ) reported previous low back pain which had required nonsurgical intervention and had resulted in time off training, five subjects (mean age 22.0 (1.8) years, mean weight 88.7 (6.9) $\mathrm{kg}$ ) reported current low back pain preventing full training, and four had no history of low back pain (mean age 21.0 (2.2) years, mean weight $83.4(3.2) \mathrm{kg}$ ).

\section{Imaging}

Subjects were scanned using a General Electric Signa SP10 interventional magnetic resonance imaging (MRI) scanner (Milwaukee, Wisconsin, USA). This is an open configuration MRI scanner consisting of two connected but opposing ring "doughnut" magnets. The gap between these magnets is 56 $\mathrm{cm}$ generating a uniform field of $0.5 \mathrm{~T}$. A transmit receive flexible coil was secured around the subject's waist and lumbar spine, and a multicoil magnetic resonance tracking device was positioned in line with the subject's lumbar spinous processes. Subjects were scanned with an FSPGR sequence. The parameters set were: time of repetitions (TR) 14.6; time of excitations (TE) 7.3; scan time two seconds; flip angle $60^{\circ}$; thickness, $10 \mathrm{~mm}$; field of view (FOV) $30 \mathrm{~cm}$; matrix $256 \times$ 


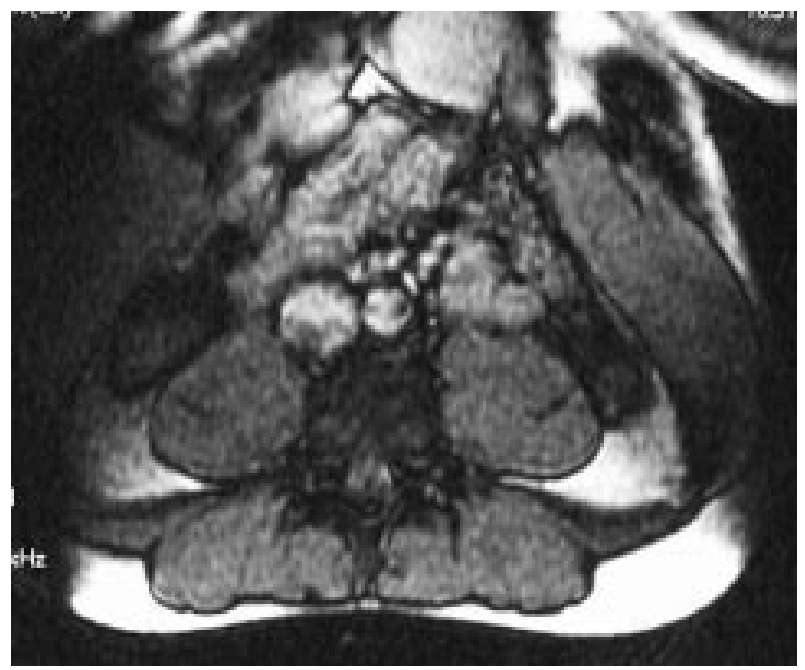

Figure 1 Axial view of the lumbar muscles at the L4-5 intervertebral junction during a contracted magnetic resonance imaging scan.

128; number of excitations 1 . This was performed in conjunction with the magnetic resonance tracking programme (General Electric) via a Sun SPARC workstation (Sun Microsystems Corporation, Mountain View, California, USA), which permitted the subject's spine to be tracked within the scanner. ${ }^{27}$

\section{Protocol}

An MRI compatible wooden rowing jig was constructed which permitted the simulation of four key stages in the rowing stroke (the catch, early and late drive, and the finish) within the scanner. ${ }^{23}$ This study focused on simulation of the catch position within the scanner. Subjects were asked to adopt their usual position at the catch phase of the stroke, the length of the oar was adjusted accordingly, and they were asked to pull on the oar (thus loading the spine and contracting the muscles) as they would while rowing. With the rower in this position, a sagittal scan of the lumbar spine was performed to localise the region of interest followed by a series of axial scans through the intervertebral junction of the L4-5 disc interspace and the L5-SI disc interspace. Subjects were asked to remain as still as possible during scanning.

\section{Image analysis}

Images were analysed on a conventional workstation to allow the measurement of muscle cross sectional area. Previous studies have shown a good correlation between MRI measures of cross sectional area and anatomical measurement. ${ }^{28}{ }^{29}$ Muscles assessed were the multifidus, erector spine muscle group at the L4-5 and L5-S1 level, and the iliopsoas at the L4-5 level.

\section{Statistical analysis}

The statistical analysis was performed using the statistical package Stata, version 6 (Stata Corporation, College Station, Texas, USA) on a personal computer. A two way analysis of variance was used to investigate if any differences existed between the three population groups. A series of covariates were considered in the analysis of variance in terms of their influence on cross sectional area, including effects of age, stroke side, and side of the body. The statistical threshold was set at $\mathrm{p}<0.05$. Orthogonal contrasts and multiple regression analysis were then used to locate where any differences noted by the analysis of variance lay.

\section{RESULTS}

The imaging protocol resulted in clear images of the lumbar spine muscles, including the erector spinae, multifidus, and
Table 1 Measures of cross sectional area $\left(\mathrm{mm}^{2}\right)$ in each of the muscle groups considered

\begin{tabular}{llll}
\hline & No LBP & Current LBP & Previous LBP \\
\hline $\begin{array}{l}\text { Multifidus L4 } \\
\text { Left }\end{array}$ & $226.3(35.0)$ & $352.4(108.8)$ & $422.2(196.60$ \\
$\begin{array}{l}\text { Right } \\
\text { Multifidus L5 }\end{array}$ & $235.5(48.7)$ & $371.0(104.1)$ & $426.3(186.3)$ \\
$\quad$ Left & $271.8(70.5)$ & $371.4(127.8)$ & $425.7 \pm 197.6)$ \\
$\quad$ Right & $277.5(87.5)$ & $362.4(101.6)$ & $428.0(218.0)$ \\
$\begin{array}{l}\text { Erector spinae L4 } \\
\text { Left }\end{array}$ & $745.0(213.9)$ & $889.4(105.8)$ & $931.6(399.1)$ \\
$\quad$ Right & $751.3(205.0)$ & $915.4(181.9)$ & $889.2(459.3)$ \\
Erector spinae L5 & $507.5(178.1)$ & $500.6(258.1)$ & $484.7(255.7)$ \\
$\quad$ Left & $545.0(153.6)$ & $515.8(224.7)$ & $440.1(209.4)$ \\
$\quad$ Right & & & \\
$\begin{array}{l}\text { lliopsoas } \\
\text { Left }\end{array}$ & $993.3(254.8)$ & $1347.8(457.2)$ & $1343.9(345.9)$ \\
Right & $916.3(207.0)$ & $1348.8(399.0)$ & $1398(407)$ \\
\hline $\begin{array}{l}\text { All measurements are mean (SD). } \\
\text { LBP, Low back pain. }\end{array}$ & & \\
& & &
\end{tabular}

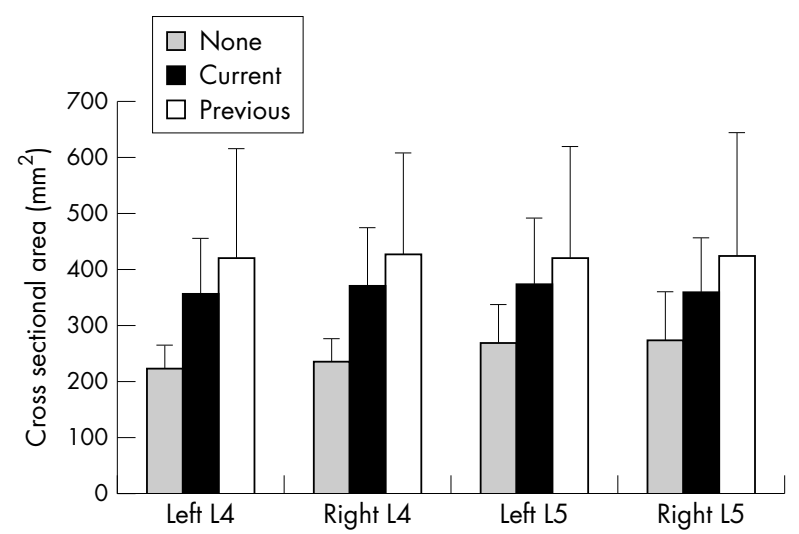

Figure 2 Cross sectional area $\left(\mathrm{mm}^{2}\right)$ of the multifidus muscle in rowers with no, current, or previous low back pain.

iliopsoas ( fig 1), from which measures of cross sectional area could be obtained.

Table 1 summarises the measurements of cross sectional area obtained on both the left and right sides of the spine.

Statistical analysis revealed significant differences with respect to cross sectional area between the three study populations. In terms of the multifidus muscle, rowers with back pain (both current and previous) were noted to have significantly larger muscles than those without back pain, this being most prominent in those with a previous history of low back pain $(p<0.0001)$ (fig 2$)$. Significant differences were also observed between subjects with a current history and those with a previous history of low back pain $(\mathrm{p}<0.001)$.

As with the multifidus muscle, significant differences were observed between the three groups when the cross sectional area of the erector spinae muscle was considered. At the L4-5 level, subjects with low back pain (both current and previous) had significantly larger muscles than those with no history of low back pain $(\mathrm{p}<0.001)$ (fig 3$)$. No differences were observed between subjects with current and those with previous low back pain. However, at the L5-S1 level, these differences were reversed, with a slight tendency for those with no history of low back pain to have larger muscles. This trend was significant when subjects with no history of low back pain were compared with those with a previous history of low back pain $(\mathrm{p}<0.05)$

When the iliopsoas muscle was considered, a similar trend was observed, with subjects with a history of low back pain (both current and previous) having larger cross sectional areas 


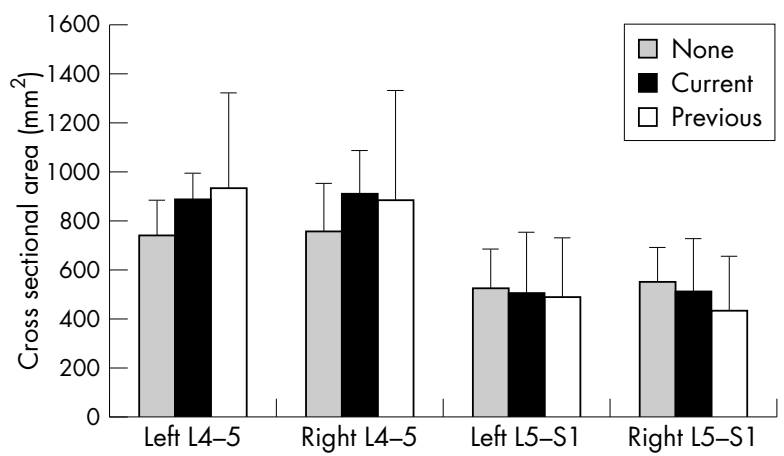

Figure 3 Cross sectional area $\left(\mathrm{mm}^{2}\right)$ of the erector spinae muscle group in rowers with no, current, or previous low back pain.

$(\mathrm{p}<0.0001)$. However, no differences were observed between the two back pain groups. When the ratio of total back extensor muscle-that is, erector spinae and multifidus-to iliopsoas cross sectional area was compared, subjects with a previous history of low back pain had a significantly lower ratio than those with no history of low back pain $(\mathrm{p}<0.05)$.

Muscle cross sectional area was influenced by age, with a decrease in muscle size with an increase in age. In some rowers, there appeared to be left and right asymmetries. However, this was not the global trend, and no significant left/right asymmetries were observed for any of the muscle groups. Similarly there were no significant asymmetries observed when oarside and non-oarside were compared.

\section{DISCUSSION}

Controversy exists about the strength of the trunk muscles and their association with both the presence and incidence of low back pain. Some studies show little or no association between strength and low back pain, ${ }^{19}{ }^{20}$ whereas others show notable weakness of the trunk muscles. ${ }^{162122}$ BieringSorensen $e a^{30}$ reported that weak trunk muscle is one of the greatest risk indicators for a first experience of low back pain, although more recent studies have disagreed with this. ${ }^{31}$ Despite this controversy, exercises to strengthen trunk muscles are commonly recommended not only as a treatment for low back pain, but as a possible preventive measure. ${ }^{32}$

Little is known about the capabilities of the trunk muscles of athletes, and the role of these muscles in the generation of low back pain. Many advocate that the treatment of athletes with low back pain should include exercises to correct imbalances and weakness. ${ }^{33}$ Muller $e t$ al ${ }^{34}$ noted that elite rowers had greater isometric torque of the trunk muscles than both control subjects and other sportspeople such as swimmers and tennis players. However, this is in contrast with the finding of Parkin et al, ${ }^{17}$ who noted no differences between elite rowing and control populations. Investigations of strength in the rowing population have not been performed.

This study has investigated measures of cross sectional area of the back muscles in a group of elite rowers with and without low back pain. Measures of cross sectional area have often been used to determine the force generating capacity of a muscle group. ${ }^{35}$ Comparisons of these three groups of rowers produced some unexpected results and suggested that rowers with back pain have larger back muscles, and thus greater strength, than rowers without low back pain. This is in contrast with the findings for the general low back pain population. It is not known if this observation is a cause or an effect of low back pain. In addition, the comparatively small study population may have influenced the findings of the study. The increased strength in the spinal muscles may be a consequence of poor technique, the rowers with back pain predominantly using their backs to generate force during the stroke rather than their legs. Previous studies have suggested that

\section{Take home message}

Low back pain in elite oarsmen does not appear to be the result of weakness or asymmetry of the multifidus or erector spinae muscle group.

there may be an alteration in the lumbopelvic rhythm of rowers with back pain that may lead to hypertrophy of the back muscles. ${ }^{19}$ It has also been proposed that patients with low back pain present with deficits in motor control as opposed to impairments in strength ${ }^{36}{ }^{37}$; if so, these changes in strength may be irrelevant. Other authors have speculated that it is fatigue not strength of the back muscles that is important. ${ }^{4}$ This was not addressed in this study.

Muller $e t a l^{34}$ noted that the extension/flexion ratio of the trunk was lower in subjects with higher rowing performance, suggesting high levels of trunk flexor strength. The influence of injury on this ratio was not assessed, because the study was unable to assess the abdominal mechanism. Consequently changes in the strength of the transversus abdominus, an important spinal stabilising muscle, have not been examined. Assessment of the cross sectional area of this muscle may provide further information to account for the unexpected finding of increased strength in the posterior stabilising muscles of the rowers with low back pain.

The asymmetric nature of rowing has been speculated to lead to the development of muscle asymmetries in the spine. Parkin $e t a l^{17}$ investigated muscle activity patterns during isometric trunk extension and noted left and right asymmetries in the rowers. However, we were unable to detect any differences in cross sectional area between the left and right sides. Similarly no differences were detected when oarside and non-oarside muscles were compared.

\section{Authors' affiliations}

A H McGregor, Department of Musculoskeletal Surgery, Imperial College School of Medicine, Charing Cross Hospital, London, UK L Anderton, W M W Gedroyc, Interventional MRI Unit, St Mary's Hospital, London, UK

\section{REFERENCES}

1 Roy SH, De Luca CJ, Casavant DA. Lumbar muscle fatigue and chronic lower back pain. Spine 1989;14:992-1001.

2 Green ACE. The prevalence of injuries in rowers. BSc thesis, Anglo-European College of Chiropractic, Portsmouth, 1998.

3 Hickey GJ, Fricker PA, McDonald WA. Injuries to elite rowers over a ten year period. Med Sci Sports Exerc 1997;29:1567-72.

4 Roy SH, De Luca CJ, Snyder-Mackler L, et al. Fatigue, recovery, and low back pain in varsity rowers. Med Sci Sports Exerc 1990;22:464-9.

5 Budgett RG, Fuller GN. Illness and injury in international oarsmen. Clin Sports Med 1989;1:55-61.

6 Stallard MC. Backache in oarsmen. Br J Sports Med 1980;14:105-8.

7 Christiansen E, Kanstrup IL. Increased risk of stress fractures of the ribs in elite rowers. Scand J Med Sci Sports 1997;7:49-52.

8 Hosea TM, Boland AL, McCarthy K, et al. Rowing injuries. Postgraduate Advances in Sports Medicine 1989;3:1-16.

9 Wajswelner H. Prevention of low back pain in rowers. Part 1: Stretching and strengthening. Excel 1987;4:10.

10 Howell DW. Musculoskeletal profile and incidence of musculoskeletal injuries in lightweight women rowers. Am J Sports Med $1984 ; 12: 278-82$.

11 Hides JA, Stokes M, Saide M, et al. Evidence of lumbar multifidus muscle wasting ipsilateral to symptoms in patient with acute/subacute low back pain. Spine 1994;19:165-72.

12 Cholewicki J, McGill SM. Mechanical stability of the in vivo lumbar spine: implications for injury and chronic low back pain. Clin Biomech 1996;1 1:1-15.

13 Quint U, Wilke H, Shiraizi-Adl A, et al. Importance of intersegmental trunk muscles for the stability of the lumbar spine. Spine 1998:23: 1937-45.

14 Panjabi M, Abumi K, Duranceau J, et al. Spinal stability and intersegmental muscle forces: a biomechanical model. Spine 1989; 14:194-200.

15 Hides JA, Richardson CA, Jull GA. Multifidus muscle recovery is not automatic after resolution of acute, first-episode low back pain. Spine 1996;21:2763-9. 
16 Rissanen A, Kalimo $\mathrm{H}$, Alaranta $\mathrm{H}$. Effect of intensive training on the isokinetic strength and structure of lumbar muscles in patients with chronic low back pain. Spine 1995;20:333-40.

17 Parkin S, Nowicky AV, Rutherford OM, et al. Do sweep stroke oarsmen have asymmetries in the strength of their back and leg muscles? J Sport Sci 2002;in press.

18 Parnianpour $M$, Nordin M, Kahanovitz N, et al. The triaxial coupling of torque generation of trunk muscles during isometric exertions and the effect of fatiguing isoinertial movements on the motor output and movement patterns. Spine 1988:13:982-92.

19 Lee JH, Hoshino Y, Nakamura K, et al. Trunk muscle weakness as a risk factor for low back pain. Spine 1999;24:54-7.

20 Lee JH, Ooi Y, Nakamura K. Measurement of muscle strength of the trunk and the lower extremities in subjects with history of low back pain. Spine 1995:20: 1994-6.

21 Takemasa R, Yamamoto $\mathrm{H}$, Tani T. Trunk muscle strength in and effect of trunk muscle exercises for patient with chronic low back pain. Spine 1995;20:2522-30

22 Hupli $M$, Hurri $\mathrm{H}$, Luoto $\mathrm{S}$, et al. Isokinetic performance capacity of trunk muscles Part I: The effect of repetition on measurements of isokinetic performance capacity of trunk muscles among healthy controls and two different groups of low back pain patients. Scand J Rehabil Med 1996;28:201-6.

23 McGregor AH, Anderton L, Gedroyc WMW. The assessment of intersegmental motion and pelvic tilt in elite oarsmen using interventional magnetic resonance imaging. Med Sci Sports Exerc 2002; in press.

24 Knapik JJ, Bauman $\mathrm{CL}$, Jones $\mathrm{BH}$, et al. Pre-season strength and flexibility imbalances associates with athletic injuries in female collegiate athletes. Am J Sports Med 1990;19:76-81.

25 Marshall JL, Tischler HM. Screening for sports: guidelines. N Y State J Med 1978;78:243-51

26 Campbell DE, Wayne G. Foot pounds of torque of the normal knee and the rehabilitated postmeniscectomy knee. Phys Ther 1979;59:418-21.
27 Dumoulin CL. Active visualization: MR tracking. In: Debatin JF, Adam $G$, eds. Interventional magnetic resonance imaging. Berlin: Springer-Verlag, 1998:65-76.

28 Beneke R, Neverburg J, Bohndorf K. Muscle cross sectiona measurement by magnetic resonance imaging. Eur J Appl Physiol $1991 ; 63: 424-9$.

29 Engstrom CM, Loeb GE, Reid JG, et al. Morphometry of the human thigh muscles, a comparison between anatomical sections and computer tomographic and magnetic resonance images. J Anat 1991;176:13956.

30 Biering-Sorensen $\mathbf{F}$, Thomsen CE, Hilden J. Risk indicators for low back trouble. Scand J Rehabil Med 1989;21:151-7.

31 Newton $M$, Thow M, Somerville D, et al. Trunk strength testing with iso-machines. Part 2: Experimental evaluation of the Cybex II Back testing system in normal subjects and patients with chronic low back pain. Spine 1993; 18:812-24

32 Gundewall B, Lilirqvist $M$, Hansson T. Primary prevention of back symptoms and absence from work: a prospective randomised study among hospital employees. Spine 1993;18:587-94.

33 D'Hemecourt PA, Gerbion PG, Micheli LJ. Back injuries in the young athlete. Clin Sports Med 2000;19:663-79.

34 Muller G, Hille E, Szpalski M. Function of the trunk musculature in elite rowers. Sportverletz Sportschaden 1994:8:134-42.

35 Clague JE, Roberts N, Gibson H, et al. Muscle imaging in health and disease. Neuromusc Disord 1995;5:171-8

36 Jull GA, Richardson CA. Motor control problems in patients with spinal pain: a new direction for therapeutic exercise. J Manipulative Physiol Ther 2000;23(2): 115-17.

37 Hodges PW, Richardson CA. Delayed postural contraction of transversus abdominus in low back pain associated with movement of the lower limb. J Spinal Disord 1998;11:45-6.

\section{Addendum}

\section{CONTEMPORARY CLASSIFICATIONS OF CONCUSSION SEVERITY AND SHORT TERM NEUROPSYCHOLOGICAL OUTCOME}

A D Hinton-Bayre', G M Geffen'. 'School of Applied Psychology, Griffith University, Gold Coast and ${ }^{2}$ Cognitive Psychophysiology Laboratory, University of Queensland, Brisbane, Queensland, Australia

Objective: To contrast concussion severity classifications in terms of subsequent impairment and recovery as shown by standard neuropsychological tests.

Setting: Prospective referral based neuropsychological assessment.

Patients: 21 professional rugby league players with a clinical diagnosis of concussion. Another 21 non-head injured players matched on key variables acted as controls to adjust for repeated assessment.

Method: All players were assessed before the season. After concussion, players were retested within 48 hours and then again at 10 days. Controls were also assessed on this schedule. For each concussed player, a grade of severity was assigned according to the Cantu (1986), Colorado Medical Society (1991), and American Academy of Neurology (1997) guidelines.
Main outcome measures: Neuropsychological outcome was indexed through subjective reports of concussion related symptoms, and performance on measures of information processing speed, including the Digit Symbol, Symbol Digit, and Speed of Comprehension tests.

Main results: Irrespective of the classification system used, concussion severity was not related to subsequent neuropsychological outcome. Several concussed players with grade 1 injuries had impaired cognitive function at 10 days, whereas those with more severe grade 3 concussions showed full recovery at the same time after trauma. Subjective reports of symptoms were clear before cognitive status in most cases. Even when concussed players had returned to the levels determined before the season, they were still relatively impaired compared with uninjured controls.

Conclusions: Contemporary classifications of concussion severity did not predict short term cognitive status. Individualised objective assessment of players is recommended to assist return to play decisions. It is important to control for practice effects, as failure to do so may underestimate the effects of the injury.

This abstract was omitted from the published abstracts of the International Symposium on Concussion in Sport $(\mathrm{Br} J$ Sports Med 2001;35:367-77). 